\title{
Quantitative Prediction of CYP3A4 Induction: Impact of Measured, Free, and Intracellular Perpetrator Concentrations from Human Hepatocyte Induction Studies on Drug-Drug Interaction Predictions ${ }^{\text {ฐ }}$
}

\author{
Yongkai Sun, Paresh P. Chothe, Jennifer E. Sager, Hong Tsao, Amanda Moore, Leena Laitinen, \\ and Niresh Hariparsad
}

Drug Metabolism and Pharmacokinetics, Vertex Pharmaceuticals Inc., Boston, Massachusetts

Received February 15, 2017; accepted March 21, 2017

\begin{abstract}
Typically, concentration-response curves are based upon nominal inducer concentrations for in-vitro-to-in-vivo extrapolation of CYP3A4 induction. The limitation of this practice is that it assumes the hepatocyte culture model is a static system. We assessed whether correcting for: 1) changes in perpetrator concentration in the induction medium during the incubation period, 2) perpetrator binding to proteins in the induction medium, and 3) nonspecific binding of perpetrator can improve the accuracy of CYP3A4 induction predictions. Of the seven compounds used in this evaluation, significant parent loss and nonspecific binding were observed for rifampicin (29.3-38.3\%), pioglitazone (64.3-78.6\%), and rosiglitazone $(57.1-75.5 \%)$. As a result, the free measured $\mathrm{EC}_{50}$ values $\left(\mathrm{EC}_{50 u}\right)$ of pioglitazone, rosiglitazone, and rifampicin were significantly lower than the nominal $\mathrm{EC}_{50}$ values. In general, the accuracy of
\end{abstract}

the induction predictions, using multiple static models, improved when corrections were made for measured medium concentrations, medium protein binding, and nonspecific binding of the perpetrator, as evidenced by $18-29 \%$ reductions in the root mean square error. The relative induction score model performed better than the basic static and mechanistic static models, resulting in lower prediction error and no false-positive or false-negative predictions. However, even when the $\mathrm{EC}_{50 \mathrm{u}}$ value was used, the induction prediction for bosentan, which is a substrate of organic anion transporter proteins, was overpredicted by approximately 2 -fold. Accounting for the ratio of unbound intracellular concentrations to unbound medium concentrations ( $\left.K_{\text {puu,in vitro }}\right)(0.5-7.5)$ and the predicted multiple-dose $K_{\text {puu,in vivo }}(0.6)$ for bosentan resulted in induction predictions within $35 \%$ of the observed interaction. introduction

Drug-drug interactions (DDIs) related to CYP3A4 induction remain a concern for the pharmaceutical industry given that induction can affect the safety and efficacy of coadministered drugs (Lin, 2006; Zhang et al., 2014). Typically, the concentration-response data used in these models rely on the nominal concentration of the applied new chemical entities (NCEs). Yet this approach is only appropriate if it can be assumed that the cell culture system is static, which may not always be the case. To address the problem of inducer depletion in vitro, various approaches have been taken into account for the changes that occur in the hepatocyte system over the course of an induction study. Kato et al. (2005) predicted the average unbound concentrations in primary cultured human hepatocyte incubations from in vivo clearance data to estimate the $\mathrm{EC}_{50}$ values used to predict induction risk. Using this method, the predicted magnitudes of induction for multiple inducers, including rifampicin and phenobarbital, were in agreement with the clinically observed DDI. In another study, Zhang et al. (2014) considered the stability of the inducers that were evaluated. However, the inducer concentration was only measured at the 24-hour time point on the last day of incubation, which may result in an overestimation of the induction potency if there

https://doi.org/10.1124/dmd.117.075481

S This article has supplemental material available at dmd.aspetjournals.org. is substantial inducer depletion during the dosing interval. Barring these assessments, metabolic changes in the in vitro cultured human hepatocyte system are typically not taken into consideration when attempting to quantitatively predict induction-based DDIs (Fahmi et al., 2008; Almond et al., 2009; Kozawa et al., 2009).

In addition to accounting for perpetrator loss, Chang et al. (2016) factored in specific binding to induction medium protein as well as nonspecific binding to assay culture plates and hepatocytes. The measured rifampicin concentration, accounting for nonspecific binding and inducer depletion over the 24-hour dosing interval, was found to be half of the nominal concentration. Furthermore, when binding to incubation medium containing $0.2 \%$ bovine serum albumin (BSA) was determined, the measured unbound inducer concentration was reportedly only $30 \%$ of the nominal level (Chang et al., 2016). Such substantial differences between nominal and measured unbound concentrations of rifampicin, which is considered to be a fairly soluble compound with moderate plasma and medium protein binding properties, suggest that, for more highly bound compounds, induction data may be drastically altered depending on whether binding is considered. This issue is important given that some compounds have very poor chemical properties which increase their risk of binding nonspecifically to assay culture plates or to proteins included in medium supplements (Rebeski et al., 1999). For example, in a recent publication of small-molecule kinase inhibitors approved by the Food and Drug Administration

ABBREVIATIONS: AUC, area under the curve; AUCR, area under the curve ratio; BSA, bovine serum albumin; CDP, XXX; DDI, drug-drug interaction; GAPDH, glyceraldehyde-3-phosphate dehydrogenase; GMFE, geometric mean fold error; MSM, mechanistic static model; NCE, new chemical entity; RED, rapid equilibrium dialysis; RIS, relative induction score; RMSE, root mean square error; TDI, time-dependent inhibition. 
from 2000 to 2011, over $70 \%$ of these drugs had plasma protein-binding values that were $>90 \%$, and $\sim 30 \%$ of them had plasma protein-binding values $>99 \%$ (O'Brien and Fallah Moghaddam, 2013). The medium unbound fraction is also likely to vary between culture systems since it is known that induction media obtained from different sources can have different types and concentrations of protein (Runge et al., 2000; Madan et al., 2003; Nishimura et al., 2007).

Given the lack of a comprehensive assessment of the relevance of using measured and unbound medium concentrations of perpetrator in induction predictions, we sought to evaluate the contribution of these factors using several static approaches, including the relative induction score (RIS), basic static $\left(\mathrm{R}_{3}\right)$, and mechanistic static [area under the curve (AUC) ratio (AUCR)] models. Seven known clinical inducers and noninducers of CYP3A4 were selected for this analysis, all of which are commonly used as calibrator compounds in the RIS model (Fahmi et al., 2008). The objectives of our study were to measure: 1) perpetrator depletion over the assay incubation period, 2) direct binding of perpetrator to proteins included in the induction medium, and 3) nonspecific binding of perpetrator to assay culture plates and hepatocytes which were then used to determine whether incorporating these corrections improves the precision and accuracy of CYP3A4 induction predictions. Finally, given that it is the perpetrator intracellular concentration that is most relevant for induction potency, we also estimated the ratio of unbound intracellular concentrations to unbound medium concentrations $\left(\mathrm{K}_{\text {puu,invitro }}\right)$ for two transporter substrates, rifampicin and bosentan, and determined the impact of using free intracellular concentrations on induction predictions.

\section{Materials and Methods}

Induction-certified cryopreserved human hepatocytes (Supplemental Table 1, Lot numbers NON, CDP, and Hu 1624) and cryopreserved 5-donor pooled hepatocytes were purchased from Thermo Fisher (Waltham, MA) or BioreclamationIVT (Baltimore, MD). Williams' E medium, cryopreserved hepatocyte recovery medium, hepatocyte maintenance and plating supplement pack, rapid equilibrium dialysis (RED) 96-well blocks, RNAlater solution, MagMax-96 Total RNA Isolation Kit, Applied Biosystems High Capacity cDNA Reverse Transcription Kit, TaqMan Fast Advanced Master Mix, TaqMan human glyceraldehyde-3-phosphate dehydrogenase (GAPDH) probe with Applied Biosystems VIC dye, and CYP3A4 cDNA probe with Applied Biosystems FAM dye were purchased from Thermo Fisher. Carbamazepine, phenytoin, pioglitazone, pleconaril, rifampicin, and rosiglitazone were purchased from Sigma-Aldrich (St. Louis, MO). Semagacestat was procured from AstaTech, Inc. (Bristol, PA). Bosentan was purchased from AvaChem Scientific (San Antonio, TX). $\left[{ }^{3} \mathrm{H}\right]$ Rifampicin $(45 \mathrm{Ci} / \mathrm{mmol})$ and $\left[{ }^{3} \mathrm{H}\right]$ bosentan $(3.3 \mathrm{Ci} / \mathrm{mmol})$ were obtained from Moravek Biochemicals (Brea, CA). LumaPlate and Ultima Gold scintillation fluid were purchased from PerkinElmer (Waltham, MA). The analytical internal standard was synthesized at Vertex Pharmaceuticals Inc. (Boston, MA). All organic solvents were purchased from Thermo Fisher and Sigma-Aldrich.

\section{CYP3A4 Induction Assay}

Cryopreserved human hepatocytes from three donors (Supplemental Table 1) were thawed in cryopreserved hepatocyte recovery medium, resuspended in Williams' E medium containing hepatocyte plating supplement pack and $10 \%$ bovine serum, and plated in collagen-coated plates at a density of $0.08 \times 10^{6}$ viable cells/well in 96-well format. After 6 hours of incubation with $5 \% \mathrm{CO}_{2}$ and $95 \%$ relative humidity at $37^{\circ} \mathrm{C}$, the plating medium was removed and replaced with the induction medium (Williams' E medium containing hepatocyte maintenance supplement pack, containing $0.125 \%$ BSA). After plating (24 hours), hepatocyte monolayers were treated with carbamazepine $(0.01-250 \mu \mathrm{M})$, phenytoin $(0.04-240 \mu \mathrm{M})$, pioglitazone $(0.03-200 \mu \mathrm{M})$, pleconaril $(0.01-60 \mu \mathrm{M})$, rifampicin $(0.01-100 \mu \mathrm{M})$, rosiglitazone $(1-50 \mu \mathrm{M})$, semagacestat $(0.01-$ $120 \mu \mathrm{M})$, and bosentan $(0.01-20 \mu \mathrm{M})$ in induction medium. Dosing solutions containing validation compounds were changed every 24 hours for 2 days. The concentration of dimethylsulfoxide in the culture medium was $0.1 \%$. Cultures were maintained in a $37^{\circ} \mathrm{C}$ incubator with $5 \% \mathrm{CO}_{2}$ and $95 \%$ humidity. All experiments were performed in triplicate.

\section{Relative Expression of mRNA by Reverse-Transcription Polymerase Chain Reaction}

Following the 48-hour compound treatment period, medium containing the test compound was removed, and the cells were stored in RNAlater solution at $-20^{\circ} \mathrm{C}$ until it was used for RNA isolation. RNA was isolated from cells using the MagMax-96 Total RNA Isolation Kit, according to the manufacturer's protocol. The RNA concentration and quality of each sample was determined using the ratio of UV absorbance at 260 and $280 \mathrm{~nm}$ (A260/A280) using a Nanodrop2000 spectrophotometer (Thermo Fisher). To generate cDNA, reverse transcription of $50 \mathrm{ng}$ of total RNA was carried out using the High Capacity cDNA Reverse Transcription Kit, according to the manufacturer's protocol. Quantitative polymerase chain reaction of $2 \mu \mathrm{l}$ of cDNA was performed using the TaqMan Fast Advanced Master Mix, human GAPDH, and CYP3A4 probes. All data were normalized to the expression of the housekeeping gene GAPDH $\left(\Delta \mathrm{Ct}=\mathrm{Ct}_{\mathrm{CYP}}-\mathrm{Ct}_{\mathrm{GAPDH}}\right)$. Fold change in mRNA expression over dimethylsulfoxide control was calculated $\left(\Delta \Delta \mathrm{Ct}=\Delta \mathrm{Ct}_{\text {treat }}-\Delta \mathrm{Ct}_{\mathrm{DMSO}}\right)$.

\section{Assessment of Nonspecific Binding and Stability of CYP3A4 Inducers}

Hepatocytes were plated following the same procedures as for the induction assay. All seven CYP3A4 inducers were incubated with hepatocytes $(n=3)$ at $37^{\circ} \mathrm{C}$ in the hepatocyte induction medium (containing $0.125 \% \mathrm{BSA}$ ) for 24 hours on day 1 and day 2 . This is the same time period that the compound was exposed to the cells in the induction assay, prior to subsequent medium change with fresh dosing medium. Incubation with hepatocytes was also carried out at $4^{\circ} \mathrm{C}$ to investigate the nonspecific binding of these compounds to cells and cell culture plates. The compound incubation concentrations ranged from 0.1 to $10 \mu \mathrm{M}$. Culture supernatants $(5 \mu \mathrm{l})$ were collected at $0,2,4,6$, and 24 hours after application of the dosing medium. As a control, the same incubations were carried out in cell culture plates without hepatocytes at both $37^{\circ} \mathrm{C}$ and $4^{\circ} \mathrm{C}$. Following sampling, $45 \mu \mathrm{l}$ of blank induction medium and $100 \mu \mathrm{l}$ of internal standard solution in acetonitrile were added to each sample. The samples were centrifuged at $3000 \mathrm{rpm}$ for 20 minutes, and the supernatants were analyzed using liquid chromatography-tandem mass spectrometry (LC-MS/MS) to determine the concentration of each test compound in the induction medium at each time point.

\section{Determination of the Unbound Fraction of CYP3A4 Inducers in Induction Medium and Hepatocytes}

Medium binding of compounds was determined in induction medium with $0.125 \% \mathrm{BSA}$ at final concentrations of 1 and $10 \mu \mathrm{M}$. Two hundred twenty-microliter aliquots were loaded into the donor chambers (red side) of the RED device and were dialyzed against $350 \mu 1$ of sodium phosphate buffer ( $\mathrm{pH} 7.4)$. The RED devices were sealed with a gas-permeable membrane and incubated on a shaking plate for 6 hours in a $37^{\circ} \mathrm{C}$ incubator containing $5 \% \mathrm{CO}_{2}$ and saturating humidity. After incubation, a $50-\mu 1$ aliquot was removed from the donor side of the RED device and added to $200 \mu \mathrm{l}$ of internal standard in acetonitrile and $50 \mu \mathrm{l}$ of buffer. A $50-\mu \mathrm{l}$ aliquot was removed from the receiver side of the RED device and added to $200 \mu \mathrm{l}$ of internal standard in acetonitrile and $50 \mu \mathrm{l}$ of induction medium. Samples were vortexed and centrifuged at $3000 \mathrm{rpm}$ for 20 minutes, and a $125-\mu 1$ aliquot of the supernatants was transferred to 96-well shallow-well plates for LC-MS/MS analysis.

Intracellular binding of bosentan and rifampicin was assessed using equilibrium dialysis (RED device) of hepatocyte homogenate using the same method that was used to assess medium binding. Binding was determined at concentrations of $1 \mu \mathrm{M}$ for both bosentan and rifampicin to capture the extent of binding at the median observed intracellular concentration. To prepare the homogenate, 10 lots of cryopreserved human hepatocytes were thawed in cryopreserved hepatocyte recovery medium and diluted in Hanks' balanced salt solution to a concentration of $10 \times 10^{6}$ total cells $/ \mathrm{ml}$ (Mateus et al., 2013). The suspension was heated at $95^{\circ} \mathrm{C}$ for 5 minutes, and then cells were lysed over three cycles of freezing on a dry-ice ethanol bath and thawing at $37^{\circ} \mathrm{C}$.

\section{Determination of Unbound Drug Accumulation Ratios $\left(\mathbf{K}_{\mathrm{puu}}\right)$ for Bosentan and Rifampicin}

Cryopreserved human hepatocytes were seeded according to the same protocol as for the induction assays. Two study designs were used to determine the 
intracellular and medium concentrations of rifampicin and bosentan. First, a timecourse experiment was performed in which cells were treated with a single concentration of either rifampicin $(0.1 \mu \mathrm{M})$ or bosentan $(1 \mu \mathrm{M})$ and incubated for $0.25,2,4,6,18$, and 24 hours to assess the change in $\mathrm{K}_{\text {puu }}$ over time. The concentrations were chosen to ensure that bosentan and rifampicin levels were sufficiently ( $>$ 5-fold) below reported $\mathrm{K}_{\mathrm{m}}$ values for OATP1B1and OATP1B3 transporters (Vavricka et al., 2002; Tirona et al., 2003; Treiber et al., 2007; Izumi et al., 2015). A follow-up experiment was conducted in which rifampicin or bosentan $(0.1,0.3,1,3,5,10$, and $20 \mu \mathrm{M})$ was incubated in cryopreserved human hepatocytes for 24 hours to determine the $K_{\text {puu }}$ values across the range of concentrations used in the induction studies. At the end of the treatment period, supernatant was collected, and $20 \mu \mathrm{l}$ was added to $180 \mu \mathrm{l}$ of acetonitrile containing internal standard. Cells were washed three times with Hanks' balanced salt solution prior to lysing with $100 \mu \mathrm{l}$ acetonitrile containing internal standard. The samples were analyzed by LC-MS/MS to measure the total intracellular concentration of a drug, considering a total intracellular volume of $4 \mu \mathrm{l} / 10^{6}$ cells (Reinoso et al., 2001). The unbound drug accumulation ratios $\left(\mathrm{K}_{\text {puu }}\right)$ were then determined using eq. 16.

\section{Characterizing the Uptake Clearance of Bosentan and Rifampicin in Suspension Hepatocytes}

The uptake of rifampicin and bosentan was measured in suspension cryopreserved human hepatocytes using the oil-spin method. Hepatocytes were thawed at $37^{\circ} \mathrm{C}$ and placed in cryopreserved hepatocyte recovery medium. Hepatocytes were centrifuged and then reconstituted in Krebs-Henseleit Buffer at a final density of $1 \times 10^{6}$ viable cells $/ \mathrm{ml}$. Cells were then incubated for 2.5 minutes at $37^{\circ} \mathrm{C}$ and $4^{\circ} \mathrm{C}$ (on ice) with different concentrations of rifampicin $(0.03-5 \mu \mathrm{M})$ spiked with $\left[{ }^{3} \mathrm{H}\right]$ rifampicin $\left.(1 \mu \mathrm{Ci} / \mathrm{ml})\right)$ and bosentan $(0.1-30 \mu \mathrm{M})$ spiked with $\left[{ }^{3} \mathrm{H}\right]$ bosentan $\left.(1 \mu \mathrm{Ci} / \mathrm{ml})\right)$. At the end of the incubation period, aliquots were taken and added to centrifuge tubes containing $25 \mu \mathrm{l}$ of $1 \mathrm{M}$ sucrose and $55 \mu \mathrm{l}$ of mineral oil (Hepatocyte Transporter Suspension Assay kit; Gentest, Tewksbury, MA). Cells were spun at $10,000 \mathrm{rpm}$ for 15 seconds to stop the uptake, and tubes were immediately placed on dry ice. Tubes were cut to separate the lower part containing the cell pellet and the upper part containing supernatant, which were then mixed with scintillation cocktail to measure radioactivity.

\section{LC-MS/MS Analysis}

The LC-MS/MS system, composed of Agilent 1200 Series binary pumps (Agilent Technologies, Palo Alto, CA), a PAL (LEAP) autosampler (CTC Analytics AG, Zwingen, Switzerland), and an API 5500 QTrap tandem mass spectrometer ( $\mathrm{AB}$ Sciex, Foster City, $\mathrm{CA}$ ), was used in the analysis of all unlabeled test compounds. A 5- $\mu$ l aliquot of each sample was injected into the LC-MS/MS system. High-performance liquid chromatography separation was conducted on a Unisol C18 column $(2.1 \times 30 \mathrm{~mm}, 5 \mu \mathrm{m}$; Agela Technologies, Newark, DE). Mobile phase A was $10 \mathrm{mM}$ ammonium acetate in water $(\mathrm{pH}$ 4.0 ), and mobile phase B was acetonitrile/methanol (50/50, v/v). The analytes were eluted using a gradient method from 0 to $99 \%$ mobile phase B in 0.5 minutes with a flow rate of $1.0 \mathrm{ml} / \mathrm{min}$, and $99 \%$ mobile phase B from 0.5 to 1.2 minutes with a flow rate from 1.0 to $1.8 \mathrm{ml} / \mathrm{min}$. The electrospray ionization source was operated at $5500 \mathrm{~V}$ or $-4500 \mathrm{~V}$ for positive and negative modes and $650^{\circ} \mathrm{C}$. The curtain gas, gas 1 , and gas 2 were set to 30,60 , and $70 \mathrm{psi}$, respectively. Multiple reaction monitoring MS/MS (Supplemental Table 2) was used to measure the analytes.

\section{Data Analysis}

$\mathbf{E C}_{50}$ and $\mathbf{E}_{\max }$ Determination for CYP3A4 mRNA Induction. GraphPad Prism 6.0 (GraphPad Software, La Jolla, CA) was used for data fitting. $\mathrm{EC}_{50}$ and $\mathrm{E}_{\max }$ values were determined from mRNA expression dose-response curves. The dose-response curves were fitted to a three-parameter sigmoid (Hill) model, with the baseline value of induction fixed to 1 , according to eq. 1 :

$$
\mathrm{E}=\frac{\mathrm{E}_{\max } * \mathrm{C} \gamma}{\mathrm{EC}_{50} \gamma+\mathrm{C} \gamma}
$$

Measured Concentration of Parent Drugs during Hepatocyte Incubation. The concentration versus time profiles of all compounds were measured over the 48-hour incubation period. $\mathrm{C}_{\min }$ is the measured concentration at 24 hours on day
1. The systemic exposure to drugs (AUC) was calculated by the linear trapezoidal method. $\mathrm{C}_{\text {ave }}$ was calculated according to eq. 2 :

$$
\mathrm{C}_{\mathrm{ave}}=\frac{\mathrm{AUC}}{24 \text { hours }} \text {. }
$$

Protein Binding with Induction Medium and Hepatocytes. The free fraction in media $\left(f_{u, \text { media }}\right)$ and the free fraction in the cell $\left(f_{u, c e l l}\right)$ were calculated according to the following equations:

$$
\begin{gathered}
\mathrm{f}_{\mathrm{u}, \text { media }}=\frac{\text { concentration in buffer }}{\text { total concentration in media }} \\
\text { concentration in buffer } \\
\text { total concentration in homogenized hepatocytes } \\
\mathrm{f}_{\mathrm{u}, \text { cell }}=\frac{1}{\mathrm{D} \times\left(\frac{1}{\mathrm{f}_{\mathrm{u}, \mathrm{hom}}}-1\right)+1} .
\end{gathered}
$$

The $f_{u, c e l l}$ value was determined by correcting the fraction unbound in homogenate $\left(f_{u, h o m}\right)$ by a dilution factor (D) as previously reported (Mateus et al., 2013; Riccardi et al., 2016). Based on a hepatocyte volume of $4 \mu \mathrm{l}$ for $1 \times 10^{6}$ cells (Reinoso et al., 2001), D was determined to be 25 .

Correcting the $\mathbf{E C}_{50}$ for Inducer Depletion and Medium Binding. Nominal $\mathrm{EC}_{50}$ was determined by plotting CYP3A4 mRNA expression fold induction in hepatocytes versus the nominal concentration $\left(\mathrm{C}_{0}\right)$ of the applied perpetrator compound. The measured $\mathrm{EC}_{50}$ was determined according to eq. 6 . Unbound $\mathrm{EC}_{50}\left(\mathrm{EC}_{50, \mathrm{u}}\right)$ was calculated for both nominal and measured $\mathrm{EC}_{50}$ according to eqs. 7 and 8 , respectively:

$$
\begin{aligned}
\text { measured } \mathrm{EC}_{50}=\text { nominal } \mathrm{EC}_{50} \times \frac{\mathrm{C}_{\mathrm{ave}}}{\mathrm{C}_{0}} \\
\text { unbound nominal } \mathrm{EC}_{50}=\text { nominal } \mathrm{EC}_{50} \times \mathrm{f}_{\mathrm{u} \text {,media }} \\
\text { unbound measured } \mathrm{EC}_{50}=\text { measured } \mathrm{EC}_{50} \times \mathrm{f}_{\mathrm{u} \text {,media. }}
\end{aligned}
$$

RIS Model and Correlation to In Vivo CYP3A4 Induction. The RIS model is a calibration approach to induction predictions in which the $\mathrm{EC}_{50}$ and $\mathrm{E}_{\max }$ of various inducers are combined with free plasma concentrations to estimate a relative induction score (Ripp et al., 2006). RIS values were calculated as described by eq. 9 (Ripp et al., 2006; Fahmi et al., 2008):

$$
\mathrm{RIS}=\frac{\mathrm{E}_{\max } \times \mathrm{C}_{\max , \mathrm{u}}}{\mathrm{EC}_{50}+\mathrm{C}_{\max , \mathrm{u}}}
$$

where $\mathrm{C}_{\max , \mathrm{u}}$ is the maximum unbound plasma concentration of the CYP3A4 inducer reported in the literature (Supplemental Table 3). The magnitude of the clinical DDI data (percentage decrease in AUC of midazolam) was plotted against the RIS value of the inducers. The curves were fitted to a three-parameter sigmoid (Hill) model, according to eq. 10. GraphPad Prism 6.0 was used for data fitting:

$$
\% \mathrm{DDI}=\frac{\% \mathrm{DDI}_{\max } \times \mathrm{RIS}^{\gamma}}{\mathrm{RIS}_{50}^{\gamma}+\mathrm{RIS}^{\gamma}} .
$$

Here, $\% \mathrm{DDI}_{\max }$ is the maximum \%DDI, and $\mathrm{RIS}_{50}$ is the RIS value at $50 \%$ of $\%$ $\mathrm{DDI}_{\max }$. An induction prediction was considered to be positive if the RIS value was $<0.1$ (Fahmi et al., 2008).

Basic Static Model $\left(\mathbf{R}_{3}\right)$. A basic static model $\left(\mathrm{R}_{3}\right.$ model), accounting for induction potency, maximum fold induction, and circulating inducer concentrations, was used in predictions. The $\mathrm{R}_{3}$ values were calculated using eq. 11 in accordance with the Food and Drug Administration (http://www.fda. gov/downloads/drugs/guidancecomplianceregulatoryinformation/guidances/ucm292362.pdf) draft guidance on drug-drug interactions. The value of $\mathrm{d}$ was set to 1 . An induction prediction was considered to be positive if the $\mathrm{R}_{3}$ value was $<0.9$ in accordance with the draft guidance:

$$
\mathrm{R}_{3}=\frac{1}{1+\mathrm{d} \times \frac{\mathrm{E}_{\max } \times[\mathrm{I}]}{\mathrm{E}_{50}+[\mathrm{I}]}} .
$$

Mechanistic Static Model. The mechanistic static model (MSM), or "net effect" model (Fahmi et al., 2009), was used to account for simultaneous reversible inhibition, time-dependent inhibition (TDI), and induction in both the 
liver and the intestine. The AUCR in the presence and absence of an inducer was calculated according to eq. 12 :

$$
\begin{aligned}
\text { AUCR }= & \frac{1}{\mathrm{f}_{\mathrm{m}, \mathrm{CYP} 3 \mathrm{~A} 4} \times[\mathrm{A} \times \mathrm{B} \times \mathrm{C}]+\left(1-\mathrm{f}_{\mathrm{m}, \mathrm{CYP} 3 \mathrm{~A} 4}\right)} \\
& \times \frac{1}{[\mathrm{X} \times \mathrm{Y} \times \mathrm{Z}] \times\left(1-\mathrm{F}_{\mathrm{G}}\right)+\mathrm{F}_{\mathrm{G}}}
\end{aligned}
$$

where $\mathrm{A}, \mathrm{B}$, and $\mathrm{C}$ denote $\mathrm{TDI}$, induction, and reversible inhibition in the liver, respectively. $\mathrm{X}, \mathrm{Y}$, and $\mathrm{Z}$ denote $\mathrm{TDI}$, induction, and reversible inhibition in the intestine. Subscripts $H$ and $G$ denote hepatic $(H)$ or intestinal $(G)$ values. These terms are described by the following equations:

$$
\begin{gathered}
\mathrm{A}(\text { or } \mathrm{X})=\frac{\mathrm{k}_{\mathrm{deg}, \mathrm{H}(\text { or G })}}{\mathrm{k}_{\mathrm{deg}, \mathrm{H}(\text { or G })}+\frac{[\mathrm{II}]_{\mathrm{H}(\text { or G })} \times \mathrm{k}_{\text {inact }}}{[\mathrm{I}]_{\mathrm{H} \text { (or G })}+\mathrm{K}_{\mathrm{I}}}} \\
\mathrm{B}(\text { or } \mathrm{Y})=1+\frac{\mathrm{d} \times \mathrm{E}_{\max } \times[\mathrm{I}]_{\mathrm{h}(\text { or G })}}{\mathrm{EC}_{50}+[\mathrm{I}]_{\mathrm{h}(\text { or G })}} \\
\mathrm{C}(\text { or } \mathrm{Z})=\frac{1}{1+\frac{\left[\mathrm{I}_{\mathrm{H}(\text { or G })}\right.}{\mathrm{K}_{\mathrm{I}}}} .
\end{gathered}
$$

$F_{G}$ is the fraction of the substrate that escapes extraction in the gut, and $f_{m, C Y P 3 A 4}$ is the fraction of the substrate cleared via CYP3A4-mediated metabolism. The values of $\mathrm{F}_{\mathrm{G}}$ and $\mathrm{f}_{\mathrm{m}, \mathrm{CYP} 3 \mathrm{~A} 4}$ were 0.51 and 0.9 for midazolam (Galetin et al., 2006; Einolf et al., 2014) and 0.66 and 0.92 for simvastatin, respectively (Chung et al., 2006; Obach et al., 2006). The unbound $\mathrm{C}_{\max }$ was used for [I] in both the intestine and the liver. The empirical scaling factor (d) was set to 1 for all predictions. Reversible inhibition of CYP3A4 by carbamazepine $\left(\mathrm{K}_{\mathrm{i}}=104 \mu \mathrm{M}\right)$, pioglitazone $\left(\mathrm{K}_{\mathrm{i}}=\right.$ $20 \mu \mathrm{M})$, and rifampicin $\left(\mathrm{K}_{\mathrm{i}}=10.5 \mu \mathrm{M}\right)$ was accounted for in the mechanistic static model. Additionally, TDI by pioglitazone $\left(\mathrm{K}_{\mathrm{I}}=13 \mu \mathrm{M}, \mathrm{k}_{\text {inact }}=0.013 \mathrm{~min}^{-1}\right)$ and rosiglitazone $\left(\mathrm{K}_{\mathrm{I}}=4.4 \mu \mathrm{M}, \mathrm{k}_{\text {inact }}=0.011 \mathrm{~min}^{-1}\right)$ was incorporated into the model. The $\mathrm{k}_{\mathrm{deg}, \mathrm{H}}$ and $\mathrm{k}_{\mathrm{deg}, \mathrm{G}}$ values were 0.019 hour $^{-1}$ and 0.029 hour $^{-1}$ (Fahmi et al., 2008). An AUCR of $<0.8$ was considered to indicate a positive induction risk, as suggested in the draft guidance (http://www.fda.gov/downloads/drugs/guidancecomplianceregulatoryinformation/guidances/ucm292362.pdf).

Evaluation of Model performance. To assess the prediction accuracy of each model, the root mean square error (RMSE, eq. 16) was calculated (Vieira et al., 2014). The geometric mean fold error (GMFE, eq. 17) was used to determine prediction bias (Vieira et al., 2014). Lower values of RMSE and GMFE indicate greater prediction accuracy and reduced bias, respectively:

$$
\begin{gathered}
\text { RMSE }=\sqrt{\frac{\sum(\text { predicted DDI }- \text { observed DDI })^{2}}{\mathrm{~N}}} \\
\text { GMFE }=10^{\frac{\sum \mid \log \frac{\text { predicted AUC ratio }}{\text { boserved ACC ratiol }}}{\mathrm{N}}} .
\end{gathered}
$$

Calculation of the In Vitro $\mathbf{K}_{\text {puu }}$. The ratio of unbound cell concentration to unbound medium concentration ( $\mathrm{K}_{\text {puu,in vitro }}$ ) was calculated according to eq. 18 (Mateus et al., 2013):

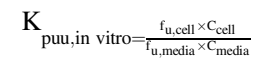

where $f_{u, c e l l}$ and $f_{u, \text { media }}$ are the fraction unbound in hepatocytes and medium, respectively. $\mathrm{C}_{\text {cell }}$ and $\mathrm{C}_{\text {media }}$ represent total intracellular and total medium drug concentration, respectively.

Correction of Bosentan and Rifampicin $\mathbf{E C}_{50}$ Values for Unbound Intracellular Concentrations. Following incubation of rifampicin and bosentan $(0.1,0.3,1,3,5,10$, and $20 \mu \mathrm{M})$ for 24 hours, intracellular and medium concentrations were measured according to the previously described protocol, and the $\mathrm{K}_{\text {puu,in vitro }}$ values were determined. The average steady-state intracellular unbound concentrations at each nominal inducer concentration $\left(\mathrm{I}_{\mathrm{u}, \text { cell,ave }}\right)$ were calculated according to eq. 19:

$$
\mathrm{I}_{\mathrm{u}, \text { cell,ave }}=\text { nominal concentration } \times\left[\frac{\mathrm{C}_{\mathrm{ave}}}{\mathrm{C}_{0}}\right] \times \mathrm{f}_{\mathrm{u} \text {,media }} \times \mathrm{K}_{\text {puu,in vitro }} .
$$

The $\mathrm{I}_{\mathrm{u} \text {,cell,ave }}$ values were plotted against the fold change in CYP3A4 mRNA, and the $\mathrm{EC}_{50 \text {,cell,u }}$ and $\mathrm{E}_{\text {max,cell }}$ values were estimated by fitting the data to eq. 1 using nonlinear regression in GraphPad Prism.
The Extended Clearance Model and Prediction of In Vivo $\mathbf{K}_{\text {puu. Hepatic }}$ blood clearance following intravenous dosing was described by the well stirred model (Pang and Rowland, 1977):

$$
\mathrm{CL}_{\mathrm{h}}=\frac{\mathrm{Q}_{\mathrm{h}} \times \mathrm{f}_{\mathrm{u}, \mathrm{b}} \times \mathrm{CL}_{\mathrm{int}, \mathrm{h}}}{\mathrm{Q}_{\mathrm{h}}+\mathrm{f}_{\mathrm{u}, \mathrm{b}} \times \mathrm{CL}_{\mathrm{int}, \mathrm{h}}}
$$

where $\mathrm{Q}_{\mathrm{h}}$ is hepatic blood flow $(90 \mathrm{l} / \mathrm{h}), \mathrm{f}_{\mathrm{u}, \mathrm{b}}$ is the unbound fraction in blood, and $\mathrm{CL}_{\mathrm{int}, \mathrm{h}}$ is the intrinsic hepatic clearance. Assuming no basolateral efflux, $\mathrm{CL}_{\mathrm{int}, \mathrm{h}}$ was defined by the extended clearance model (Liu and Pang, 2005):

$$
\mathrm{CL}_{\text {int,h }}=\left(\mathrm{CL}_{\text {int,uptake }}+\mathrm{CL}_{\text {int,passive }}\right) \times \frac{\left(\sum \mathrm{CL}_{\text {int,metab }}+\mathrm{CL}_{\text {int,bile }}\right)}{\left(\mathrm{CL}_{\text {int,passive }}+\sum \mathrm{CL}_{\text {int,metab }}+\mathrm{CL}_{\text {int,bile }}\right)}
$$

where $\mathrm{CL}_{\text {int,uptake }}$ is intrinsic active uptake clearance, $\mathrm{CL}_{\text {int,passive }}$ is the intrinsic passive diffusion clearance, $\mathrm{CL}_{\text {int,bile }}$ is the intrinsic biliary clearance, and $\mathrm{CL}_{\text {int,metab }}$ is the total intrinsic metabolic clearance. 2013):

The in vivo $\mathrm{K}_{\mathrm{puu}}\left(\mathrm{K}_{\mathrm{puu}, \text { in vivo }}\right)$ was predicted according to eq. 22 (Barton et al.,

$$
\mathrm{K}_{\text {puu,in vivo }}=\frac{\mathrm{SF}_{\text {uptake }} \times \mathrm{CL}_{\text {int,uptake }}+\mathrm{CL}_{\text {int,passive }}}{\mathrm{CL}_{\text {int,passive }}+\mathrm{CL}_{\text {int,bile }}+\mathrm{CL}_{\text {int,metab }}}
$$

where $\mathrm{SF}_{\text {uptake }}$ is an empirical scaling factor for in vitro $\mathrm{CL}_{\text {int,uptake }}$ that was estimated by comparing the in vitro $\mathrm{CL}_{\text {int,uptake }}$ to the in vivo $\mathrm{CL}_{\text {int,uptake }}$ estimated from $\mathrm{CL}_{\mathrm{h}}$. In vitro intrinsic clearance values were scaled by assuming $39.8 \mathrm{mg}$ microsomal protein/g liver, $118 \times 10^{6}$ hepatocytes/g liver, $24.5 \mathrm{~g}$ liver $/ \mathrm{kg}$ body weight, and an average $70-\mathrm{kg}$ body weight.

Induction Predictions Incorporating In Vitro and In Vivo $K_{\text {puu }}$ Estimates. Induction predictions using the $\mathrm{R}_{3}$ (eq. 11) and MSM (eq. 12) methods were performed to assess the effects of intracellular unbound concentrations on prediction accuracy. $\mathrm{E}_{\mathrm{max}, \mathrm{u} \text {,cell }}$ and $\mathrm{EC}_{50, \mathrm{u}, \text { cell }}$ were used as input parameters. The $[\mathrm{I}]$ value used for the $\mathrm{R}_{3}$ model and the hepatic component of the mechanistic static model was equal to $\mathrm{C}_{\mathrm{max}, \mathrm{u}} * \mathrm{~K}_{\mathrm{puu}}$. For the intestinal portion of the MSM model, [I] was assumed to be equal to $\mathrm{C}_{\mathrm{max}, \mathrm{u}}$.

In Vitro Uptake Kinetics Measurement. The active uptake velocity was determined by subtracting the velocity at $4^{\circ} \mathrm{C}$ from the uptake velocity at $37^{\circ} \mathrm{C}$. The active uptake velocity was plotted against substrate concentration and fit via nonlinear regression in GraphPad Prism 6.0. The kinetic parameters $K_{m}$ and $V_{\max }$ for active uptake were determined using the Michaelis-Menten equation (eq. 23). $\mathrm{CL}_{\text {int,uptake }}$ was estimated from the ratio of the $\mathrm{V}_{\max }$ and $\mathrm{K}_{\mathrm{m}}$ values:

$$
\mathrm{v}=\frac{\mathrm{V}_{\max } \times \mathrm{S}}{\mathrm{K}_{\mathrm{m}}+\mathrm{S}}
$$

Intrinsic passive permeability clearance $\left(\mathrm{CL}_{\text {int,passive }}\right)$ was estimated from the slope of the $4^{\circ} \mathrm{C}$ velocity versus substrate concentration plots using linear regression.

\section{Results}

Measured Concentration of CYP3A4 Inducers in Human Hepatocyte Induction Medium. At $37^{\circ} \mathrm{C}$, negligible parent compound loss was observed for phenytoin, carbamazepine, and semagacestat. The concentration change over 24 hours after dosing was within analytical accuracy (data not shown). The profiles of measured concentrations over time for pioglitazone, pleconaril, rifampicin, and rosiglitazone are shown in Fig. 1. Rifampicin concentration decreased when incubated at $37^{\circ} \mathrm{C}$, with $56-83 \%$ remaining at the 24 -hour time point (Fig. 1C) after dosing at three concentrations and incubating with three lots of hepatocytes. Pioglitazone (Fig. 1A) and rosiglitazone (Fig. 1D) showed substantial parent loss when incubated at $37^{\circ} \mathrm{C}$, with $\sim 20-50 \%$ remaining at the 24 hour time point after dosing. At $37^{\circ} \mathrm{C}$, the percentage of pleconaril remaining (Fig. 1B) decreased to $<10 \%$ after 24-hour incubation. No notable difference in perpetrator concentration profiles over time was observed between day 1 and day 2 (Fig. 1). The 
A Pioglitazone
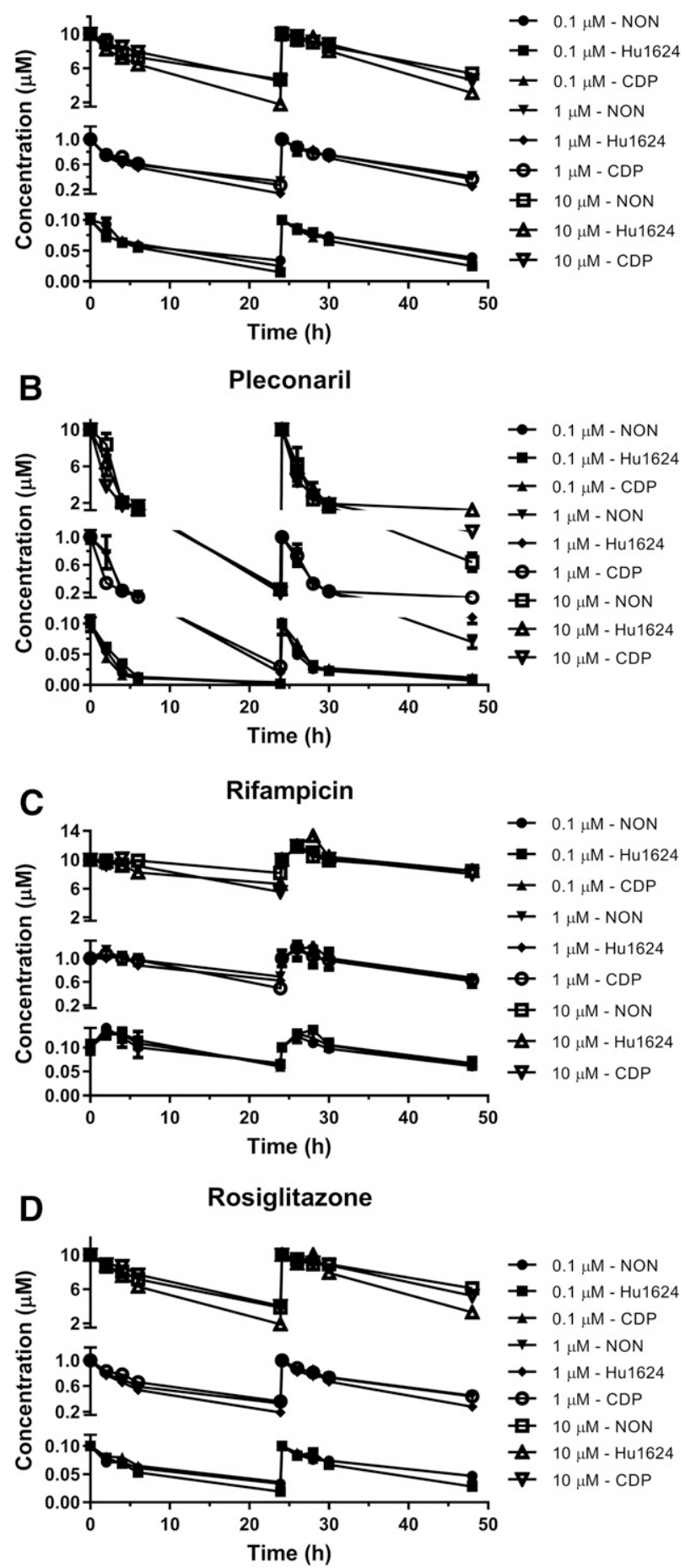

Fig. 1. Parent compound loss of various CYP3A4 inducers in human hepatocyte induction assay. Measured medium concentration of pioglitazone (A), pleconaril (B), rifampicin (C), and rosiglitazone (D) over 48-hour incubation with primary human hepatocytes at $37^{\circ} \mathrm{C}$ in $0.125 \% \mathrm{BSA}$ containing medium. The inducers were dosed at concentrations of $0.1,1.0$, and $10 \mu \mathrm{M}$ on day 1 and day 2. Data are represented as the mean \pm standard error in triplicate for three hepatocyte lots.

concentrations of pioglitazone, rifampicin, and rosiglitazone were unchanged over the 24-hour incubation period when incubated at $4^{\circ} \mathrm{C}$ (Supplemental Fig. 1, A, C, and D). However, loss of pleconaril was observed at $4{ }^{\circ} \mathrm{C}$ (Supplemental Fig. 1B). Similarly, when these compounds were incubated with cell culture plates alone (without hepatocytes; data not shown), pleconaril concentration decreased notably, but the concentrations of the other compounds were close to nominal concentrations at $37^{\circ} \mathrm{C}$ and $4{ }^{\circ} \mathrm{C}$, which indicated nonspecific binding of pleconaril to cell plates. The average measured concentration $\left(\mathrm{C}_{\mathrm{ave}}=\mathrm{AUC} /\right.$ time $)$ of each CYP3A4 inducer was calculated using the overall exposure (AUC) and incubation time interval (time), whereas minimum concentration $\left(\mathrm{C}_{\min }\right)$ was the measured concentration at the 24-hour time point. For each compound, the average percentage remaining and the percentage remaining at the 24-hour time point are summarized in Table 1.

Protein Binding of CYP3A4 Inducers in Hepatocyte Induction Medium. The hepatocyte induction medium that was used in these studies contains $0.125 \%$ BSA and other proteins, such as insulin $(6.25 \mu \mathrm{g} / \mathrm{ml})$ and transferrin $(6.25 \mu \mathrm{g} / \mathrm{ml})$. For six of the seven CYP3A4 inducers, recovery in the protein-binding assay ranged between 80 and $120 \%$ in both media (Table 1). However, the protein-binding assay for pleconaril resulted in poor recovery values, possibly due to high nonspecific binding and/or poor stability, and was therefore not reported in Table 2. Pioglitazone and rosiglitazone were highly protein bound in the induction medium, with $\mathrm{f}_{\mathrm{u}}$ values of 0.22 and 0.15 , respectively (Table 1). Carbamazepine $\left(f_{u}=0.89\right)$, phenytoin $\left(f_{u}=0.90\right)$, and semagacestat $\left(f_{u}=0.91\right)$ showed less than $11 \%$ binding.

CYP3A4 Induction and Generation of RIS Models Based on Nominal and Measured Concentrations of the Perpetrator in Hepatocyte Induction Medium. For each of the seven CYP3A4 inducers included in this study, the concentration-dependent changes in CYP3A4 mRNA expression were used to determine the $\mathrm{E}_{\max }$ and $\mathrm{EC}_{50}$ values in three donors (Table 1 ). The $\mathrm{EC}_{50}$ was subsequently corrected for the measured and free drug concentrations in the hepatocyte induction medium, as illustrated in Fig. 2. The resulting $\mathrm{EC}_{50}$ values for nominal $\left(\mathrm{C}_{0}\right)$, nominal unbound $\left(\mathrm{C}_{0, \mathrm{u}}\right)$, measured $\left(\mathrm{C}_{\mathrm{ave}}\right)$, and measured unbound $\left(\mathrm{C}_{\mathrm{ave}, \mathrm{u}}\right)$ concentrations are shown in Table 1. For four of the compounds, the measured $\mathrm{EC}_{50}$ values were equivalent to or only slightly lower than the nominal $\mathrm{EC}_{50}$ values. However, for compounds where substantial depletion and nonspecific binding were observed, the measured $\mathrm{EC}_{50}$ values were $27 \%$ (rosiglitazone), $44 \%$ (pioglitazone), and $82 \%$ (pleconaril) lower than the nominal $\mathrm{EC}_{50}$ values. Similar results were observed upon correcting the $\mathrm{EC}_{50}$ values for medium binding. Whereas the measured $\mathrm{EC}_{50, \mathrm{u}}$ values for carbamazepine, phenytoin, and semagacestat were not substantially different from the nominal $\mathrm{EC}_{50}$ values, the measured $\mathrm{EC}_{50, \mathrm{u}}$ values for more highly bound compounds were $48 \%$ (rifampicin), $87 \%$ (pioglitazone), and $90 \%$ (rosiglitazone) lower than the nominal $\mathrm{EC}_{50}$ after correction by protein binding.

Using the various $\mathrm{EC}_{50}$ values generated, four different RIS curves (nominal RIS, measured RIS, nominal RIS $\mathrm{u}_{\mathrm{u}}$, and measured RIS $\mathrm{R}_{\mathrm{u}}$ ) were calculated according to eq. 7. Representative regression curves are shown in Supplemental Fig. 2.

Observed versus Predicted DDI by RIS Modeling. The RIS curves were then used to predict the DDI potential of the seven inducers, and the results are summarized in Supplemental Table 4. The magnitude of induction predicted from all three hepatocyte donors is close to the observed, as shown in Fig. 3. Each of the four RIS models correctly identified all compounds as true positives or negatives. The RIS model utilizing nominal $\mathrm{EC}_{50}$ values predicted the AUCR to be within 2-fold of the observed for four of the seven compounds, whereas the induction magnitude for carbamazepine and phenytoin was underpredicted $(<2$ fold of the observed), and semagacestat induction was overpredicted ( $>$ 2-fold of observed). Prediction accuracy was not improved by using the RIS model that incorporated measured medium concentrations, as evidenced by an increase in GMFE and RMSE in two of the three donors (Table 2). However, accounting for protein binding in the RIS models 
TABLE 1

Nominal $\mathrm{EC}_{50}$ of CYP3A4 mRNA induction corrected for measured and unbound concentration of perpetrator in induction medium

\begin{tabular}{|c|c|c|c|c|c|c|c|c|}
\hline \multirow{2}{*}{ Hepatocyte Lot } & \multicolumn{2}{|c|}{ Stability } & \multirow{2}{*}{$\begin{array}{l}\text { Induction Medium Protein } \\
\text { Binding } \mathrm{f}_{\mathrm{u}}(\% \text { Recovery })\end{array}$} & \multirow{2}{*}{$\mathrm{E}_{\max }$} & \multicolumn{2}{|c|}{$\mathrm{EC}_{50}$} & \multicolumn{2}{|c|}{$\mathrm{EC}_{50, \mathrm{u}}$} \\
\hline & Average $\%$ Remaining ${ }^{a}$ & $\%$ Remaining at $24 \mathrm{~h}$ & & & Nominal $^{b}$ & Measured $^{c}$ & Nominal $^{d}$ & Measured $^{e}$ \\
\hline \multicolumn{9}{|l|}{ Carbamazepine } \\
\hline NON & 100 & 100 & $0.89(95.5)$ & 11 & 31 & 31 & 28 & 28 \\
\hline $\mathrm{Hu} 1624$ & 100 & 100 & & 10 & 23 & 23 & 21 & 21 \\
\hline CDP & 100 & 100 & & 5.3 & 23 & 23 & 21 & 21 \\
\hline \multicolumn{9}{|l|}{ Phenytoin } \\
\hline NON & 100 & 100 & $0.90(110)$ & 11 & 22 & 22 & 22 & 22 \\
\hline Hu1624 & 100 & 100 & & 12 & 25 & 25 & 25 & 25 \\
\hline CDP & 100 & 100 & & 7.7 & 27 & 27 & 27 & 27 \\
\hline \multicolumn{9}{|l|}{ Pioglitazone } \\
\hline NON & 62.4 & 41.3 & 0.21 (112) & 8.8 & 4.4 & 2.5 & 1 & 0.58 \\
\hline Hu1624 & 52.2 & 21.4 & & 7.7 & 11 & 6.4 & 2.5 & 1.5 \\
\hline CDP & 61 & 35.7 & & 6.9 & 21 & 12 & 4.8 & 2.8 \\
\hline \multicolumn{9}{|l|}{ Pleconaril } \\
\hline NON & 20.4 & 4.5 & ND (10.5) & 6.6 & 14 & 2.5 & ND & ND \\
\hline $\mathrm{Hu} 1624$ & 21.5 & 6.5 & & 2.9 & 2.4 & 0.43 & ND & ND \\
\hline CDP & 20.6 & 7.5 & & 2.5 & 6.6 & 1.2 & ND & ND \\
\hline \multicolumn{9}{|l|}{ Rifampicin } \\
\hline NON & 89.7 & 70.7 & $0.61(105)$ & 23 & 1.5 & 1.3 & 0.92 & 0.78 \\
\hline Hu1624 & 89.7 & 68.7 & & 12 & 0.28 & 0.24 & 0.17 & 0.15 \\
\hline $\mathrm{CDP}$ & 87.9 & 61.7 & & 14 & 0.36 & 0.31 & 0.22 & 0.19 \\
\hline \multicolumn{9}{|l|}{ Rosiglitazone } \\
\hline NON & 63.1 & 42.9 & 0.15 (111) & 17 & 15 & 11 & 2.1 & 1.5 \\
\hline $\mathrm{Hu} 1624$ & 53.3 & 24.5 & & 9.3 & 15 & 11 & 2.1 & 1.5 \\
\hline CDP & 64.7 & 42.5 & & 9.6 & 22 & 16 & 3.1 & 2.2 \\
\hline \multicolumn{9}{|l|}{ Semagacestat } \\
\hline NON & 100 & 100 & $0.91(115)$ & 23 & 2.6 & 2.6 & 2.3 & 2.3 \\
\hline Hu1624 & 100 & 100 & & 9.9 & 1.6 & 1.6 & 1.4 & 1.4 \\
\hline $\mathrm{CDP}$ & 100 & 100 & & 17 & 1.8 & 1.8 & 1.6 & 1.6 \\
\hline
\end{tabular}

$\mathrm{f}_{\mathrm{u}}$, fraction unbound; ND, not determined.

${ }^{a}$ Average \%remaining $=\mathrm{C}_{\mathrm{ave}} / \mathrm{C}_{0} \times 100 \%$.

${ }^{b}$ Nominal $\mathrm{EC}_{50}$ is the $\mathrm{EC}_{50}$ value determined based on the nominal concentration of dosing solution (total $\mathrm{C}_{0}$ ).

${ }^{c}$ Measured $\mathrm{EC}_{50}$ is the $\mathrm{EC}_{50}$ value determined based on the measured concentration in induction medium (total $\mathrm{C}_{\mathrm{ave}}$ ): measured $\mathrm{EC}_{50}=$ nominal $\mathrm{EC}_{50} \times \mathrm{C}_{\mathrm{ave}} / \mathrm{C}_{0}$.

${ }^{d}$ Nominal unbound $\mathrm{EC}_{50}\left(\mathrm{EC}_{50, \mathrm{u}}\right)$ is the $\mathrm{EC}_{50}$ value determined based on the unbound concentration of dosing solution $\left(\mathrm{C}_{0, \mathrm{u}}\right)$ : unbound $\mathrm{EC}_{50}=$ nominal $\mathrm{EC}_{50} \times \mathrm{f}_{\mathrm{u}}(\mathrm{media})$

${ }^{e}$ Measured $\mathrm{EC}_{50, \mathrm{u}}$ is the $\mathrm{EC}_{50}$ value determined based on the measured unbound concentration $\left(\mathrm{C}_{\mathrm{ave}, \mathrm{u}}\right)$ : measured $\mathrm{EC}_{50, \mathrm{u}}=$ nominal $\mathrm{EC}_{50} \times \mathrm{f}_{\mathrm{u}}($ medium $) \times \mathrm{C}_{\mathrm{ave}} / \mathrm{C}_{0}$.

notably improved the prediction accuracy. Regardless of whether protein binding was considered alone or in combination with measured medium concentrations, predictions of phenytoin induction were accurately predicted within 2-fold of the observed. Additionally, the GMFE and RMSE were lower in two of the three donors upon correction for protein binding, with the greatest accuracy (lowest RMSE) values observed when both binding and measured concentrations were included in the model (Table 2). As medium protein binding was not determined for pleconaril, predicted DDI was not determined using the unbound RIS modeling.

Static Modeling Predictions. In addition to RIS modeling, staticprediction models were also tested for their predictive ability (Supplemental Tables 5 and 6). The basic static model predicted DDI risk relatively well across the four quadrants, but use of the nominal $\mathrm{EC}_{50}$ resulted in false-negative predictions (positive induction AUCR $<0.9)$ for pioglitazone and pleconaril in all three donors (Fig. 4; Supplemental Table 5). Use of the measured $\mathrm{EC}_{50}$ as opposed to the nominal value resulted in true-positive predictions for pleconaril and a reduction in false-negative predictions from 29 to $14 \%$ of the seven predictions (Table 2). The fraction of false negatives was further reduced when medium protein binding was accounted for, with only one false-negative prediction for pioglitazone in donor CDP. Phenytoin and carbamazepine, although true positives, were consistently underpredicted ( $<2$-fold of the observed AUCR) across all three donors, whereas semagacestat was overpredicted $(>2$-fold of the observed AUCR) in donors NON and CDP, regardless of which $\mathrm{EC}_{50}$ values were used. However, RMSE values declined when the $\mathrm{EC}_{50}$ value was corrected for the average measured medium concentrations, and further decreased upon consideration of in vitro protein binding (Table 2), indicating increased prediction precision. Despite improved accuracy, prediction bias slightly increased (higher GFME) when $\mathrm{EC}_{50}$ values were corrected for protein binding (Table 2).

The last approach that was evaluated was the MSM, which takes into account $\mathrm{f}_{\mathrm{m}, \mathrm{CYP} 3 \mathrm{~A} 4}, \mathrm{~F}_{\mathrm{G}}$, and simultaneous reversible and timedependent inhibition (Fig. 5; Supplemental Table 6). The MSM also resulted in relatively accurate classifications of the compounds. Correcting the $\mathrm{EC}_{50}$ for average measured concentrations reduced the percentage of false negatives from $29 \%$ to an average of $24 \%$ across the three donors (Table 2), which is a minor improvement compared with what was observed with the $R_{3}$ model, due to differences in the cutoff values used in these two methods. Factoring protein binding into the $\mathrm{EC}_{50}$ estimate resulted in further reductions in false negatives, with the lowest incidence observed when using the measured $\mathrm{EC}_{50, \mathrm{u}}$. The magnitude of carbamazepine and phenytoin induction was underpredicted, and rifampicin and semagacestat induction was overpredicted in all three donors, regardless of which $\mathrm{EC}_{50}$ value was used. However, the RMSE was reduced when protein binding was accounted for, and was further reduced when the $\mathrm{EC}_{50}$ value was corrected for the average measured medium concentration. Very little change was seen in the GFME values, with slight increases observed upon correction for protein binding, indicating a minor increase in prediction bias.

Correction of $\mathbf{E C}_{50}$ Values for Unbound Intracellular Concentrations of CYP3A4 Inducers in Hepatocytes. Ultimately, it is the intracellular concentration of the perpetrator that drives the induction 
TABLE 2

Accuracy and bias in the prediction of clinical CYP3A4 induction using various static models

The number of false negatives for each prediction method is listed. In parenthesis is the total number of predictions in the data set. For the $\mathrm{R}_{3}$ model, an $\mathrm{R}$ value $<0.9$ is considered to indicate induction risk. For the mechanistic static, induction potential is considered possible if the AUCR value is $<0.8$.

\begin{tabular}{|c|c|c|c|c|}
\hline Lot & Nominal $\mathrm{EC}_{50}{ }^{a}$ & Measured $\mathrm{EC}_{50}{ }^{b}$ & Nominal $\mathrm{EC}_{50, \mathrm{u}}{ }^{c}$ & Measured $\mathrm{EC}_{50, \mathrm{u}}{ }^{d}$ \\
\hline \multicolumn{5}{|l|}{ RIS model } \\
\hline \multicolumn{5}{|l|}{ RMSE } \\
\hline NON & 9.09 & 6.25 & 6.25 & 6.25 \\
\hline $\mathrm{Hu} 1624$ & 7.40 & 11.40 & 6.25 & 6.25 \\
\hline $\mathrm{CDP}$ & 6.66 & 10.39 & 6.25 & 6.25 \\
\hline \multicolumn{5}{|l|}{ GMFE } \\
\hline NON & 1.58 & 1.47 & 1.52 & 1.52 \\
\hline Hu 1624 & 1.53 & 1.74 & 1.52 & 1.52 \\
\hline CDP & 1.49 & 1.68 & 1.52 & 1.52 \\
\hline \multicolumn{5}{|l|}{ False negative $(\mathrm{N})$} \\
\hline $\mathrm{NON}$ & $0(7)$ & $0(7)$ & $0(6)$ & $0(6)$ \\
\hline Hu1624 & $0(7)$ & $0(7)$ & $0(6)$ & $0(6)$ \\
\hline $\mathrm{CDP}$ & $0(7)$ & $0(7)$ & $0(6)$ & $0(6)$ \\
\hline \multicolumn{5}{|l|}{ Basic static $\left(\mathrm{R}_{3}\right)$} \\
\hline \multicolumn{5}{|l|}{ RMSE } \\
\hline NON & 20.84 & 18.35 & 16.05 & 15.88 \\
\hline Hu1624 & 19.67 & 16.53 & 16.22 & 15.43 \\
\hline CDP & 26.02 & 24.58 & 23.76 & 23.38 \\
\hline \multicolumn{5}{|l|}{ GMFE } \\
\hline $\mathrm{NON}$ & 2.16 & 2.13 & 2.29 & 2.29 \\
\hline Hu 1624 & 1.99 & 1.92 & 2.06 & 2.04 \\
\hline CDP & 2.34 & 2.30 & 2.48 & 2.46 \\
\hline \multicolumn{5}{|c|}{ False negative (N) } \\
\hline $\mathrm{NON}$ & $2(7)$ & $1(7)$ & $0(6)$ & $0(6)$ \\
\hline Hu1624 & $2(7)$ & $1(7)$ & $1(6)$ & $0(6)$ \\
\hline CDP & $2(7)$ & $1(7)$ & $1(6)$ & $1(6)$ \\
\hline \multicolumn{5}{|c|}{ Mechanistic static with inhibition } \\
\hline \multicolumn{5}{|c|}{ RMSE } \\
\hline NON & 19.64 & 15.77 & 11.99 & 11.50 \\
\hline Hu1624 & 19.29 & 15.94 & 14.97 & 13.20 \\
\hline CDP & 24.02 & 22.00 & 20.88 & 19.89 \\
\hline \multicolumn{5}{|l|}{ GMFE } \\
\hline $\mathrm{NON}$ & 2.93 & 2.98 & 3.42 & 3.46 \\
\hline Hu 1624 & 2.36 & 2.24 & 2.53 & 2.67 \\
\hline CDP & 3.19 & 3.27 & 3.79 & 3.79 \\
\hline \multicolumn{5}{|l|}{ False negative $(\mathrm{N})$} \\
\hline NON & $2(7)$ & $2(7)$ & $1(6)$ & $0(6)$ \\
\hline Hu1624 & $2(7)$ & $1(7)$ & $1(6)$ & $1(6)$ \\
\hline CDP & $2(7)$ & $2(7)$ & $1(6)$ & $1(6)$ \\
\hline
\end{tabular}

${ }^{a}$ Nominal $\mathrm{EC}_{50}$ is the $\mathrm{EC}_{50}$ value determined based on the nominal concentration of dosing solution (total $\mathrm{C}_{0}$ ).

${ }^{b}$ Measured $\mathrm{EC}_{50}$ is the $\mathrm{EC}_{50}$ value determined based on the measured concentration in induction medium (total $\mathrm{C}_{\mathrm{ave}}$ ): measured $\mathrm{EC}_{50}=$ nominal $\mathrm{EC}_{50} \times \mathrm{C}_{\mathrm{ave}} / \mathrm{C}_{0}$.

${ }^{c}$ Nominal unbound $\mathrm{EC}_{50}\left(\mathrm{EC}_{50, \mathrm{u}}\right)$ is the $\mathrm{EC}_{50}$ value determined based on the unbound concentration of dosing solution $\left(\mathrm{C}_{0, \mathrm{u}}\right)$ : unbound $\mathrm{EC}_{50}=$ nominal $\mathrm{EC}_{50} \times \mathrm{f}_{\mathrm{u}}($ medium $)$.

${ }^{d}$ Measured $\mathrm{EC}_{50, \mathrm{u}}$ is the $\mathrm{EC}_{50}$ value determined based on the measured unbound concentration in induction medium $\left(\mathrm{C}_{\mathrm{ave}, \mathrm{u}}\right)$ : measured $\mathrm{EC}_{50, \mathrm{u}}=$ nominal $\mathrm{EC}_{50} \times \mathrm{f}_{\mathrm{u}}($ medium $) \times \mathrm{C}_{\mathrm{ave}} / \mathrm{C}_{0}$

response. This becomes particularly relevant for compounds that are both substrates of uptake transporters as well as CYP3A4 inducers since it cannot be assumed that free intracellular concentrations are equal to free medium concentrations. Therefore, we used organic aniontransporting polypeptide substrates rifampicin and bosentan as model drugs (Treiber et al., 2007; Shou et al., 2008) to compare prediction success after using nominal, measured, and unbound medium concentrations for $\mathrm{EC}_{50}$ determination to the predictions resulting from the use of measured $\mathrm{EC}_{50}$ values, based on intracellular unbound concentrations $\left(\mathrm{EC}_{50, \mathrm{cell}, \mathrm{u}}\right)$. Bosentan induction was assessed in cryopreserved human hepatocytes from two donors (CDP, Hu1624) (Supplemental Table 1). The resulting $\mathrm{EC}_{50}$ values were corrected for the average percentage remaining (33-44\%) and $\mathrm{f}_{\mathrm{u} \text {,medium }}(0.377)$ (Table 3 ). In contrast to the inducer predictions previously discussed, predictive error for the $\mathrm{R}_{3}$ and MSM models was greater (93-135\%) when the $\mathrm{EC}_{50}$ values were corrected for binding and measured $\mathrm{C}_{\mathrm{ave}}$ than when nominal $\mathrm{EC}_{50}$ values were used (14-35\% error), resulting in approximately 2-fold overpredictions. One potential explanation for the observed overpredictions could be that the intracellular unbound concentrations are higher than the unbound medium concentrations. In this scenario, using the unbound medium concentrations for $\mathrm{EC}_{50}$ determination would result in an overestimation of the inducer potency. To test this hypothesis, we

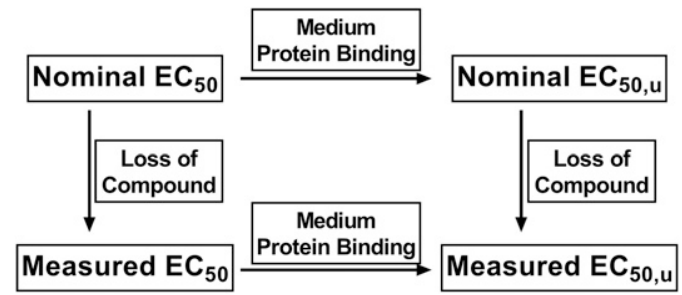

Fig. 2. Schematic for correction of in vitro induction data using measured compound concentration in induction medium and medium protein binding. Correction of nominal $\mathrm{EC}_{50}$ and RIS for loss of compound in the assay incubation results in the measured $\mathrm{EC}_{50}$ and RIS, whereas correction for medium protein binding results in nominal unbound $\mathrm{EC}_{50, \mathrm{u}}$ and $\mathrm{RIS}_{\mathrm{u}}$. Both resulting values can be further corrected by loss of compound or medium protein binding to yield measured unbound $\mathrm{EC}_{50, \mathrm{u}}$ and $\mathrm{RIS}_{\mathrm{u}}$. 

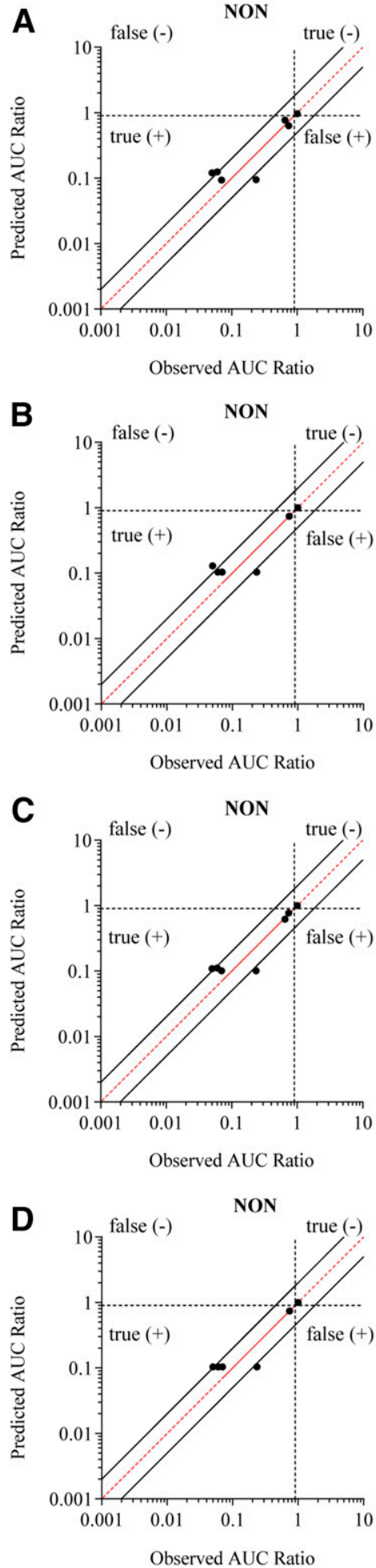

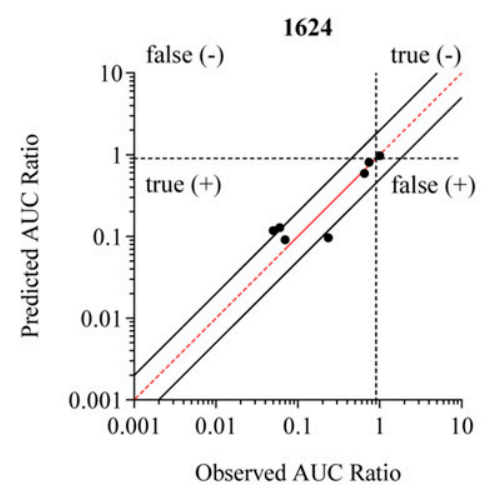

1624

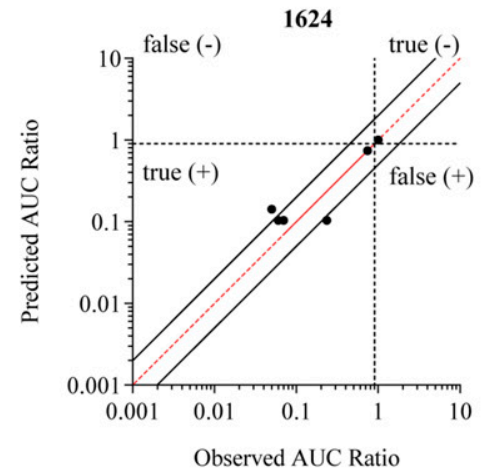

1624

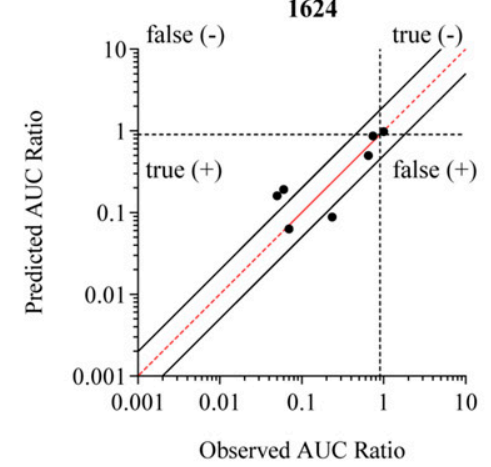

1624

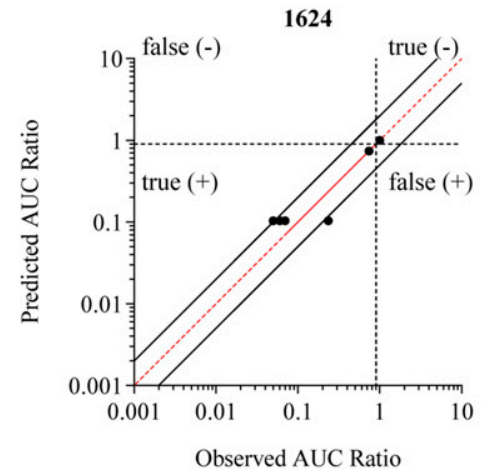

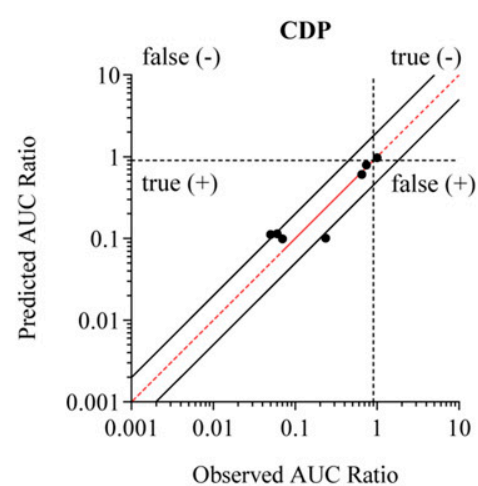

CDP

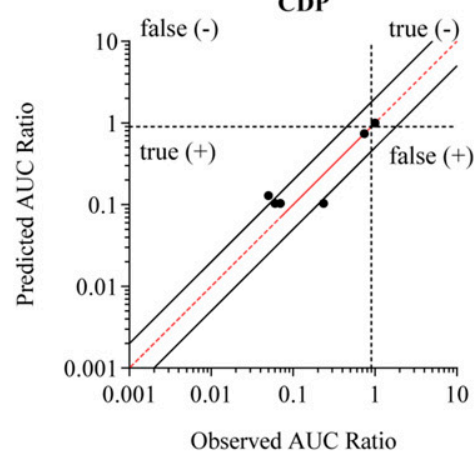

CDP

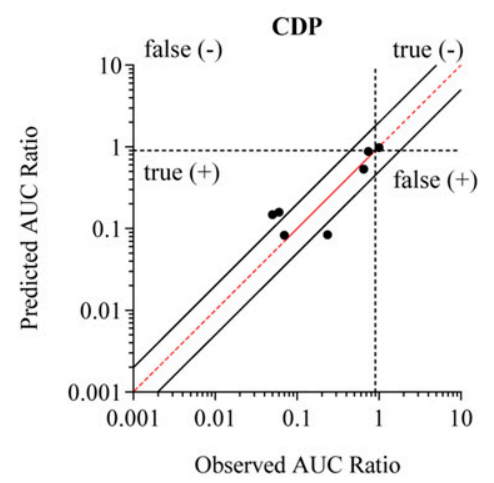

CDP

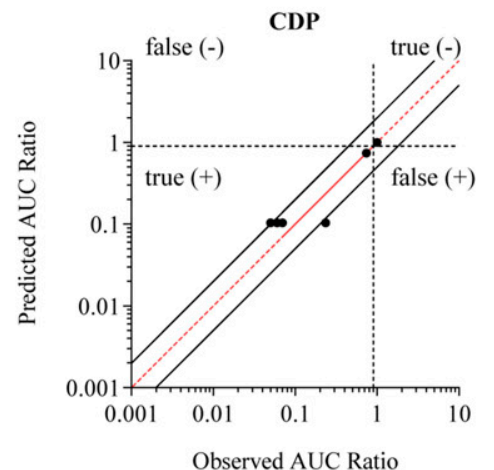

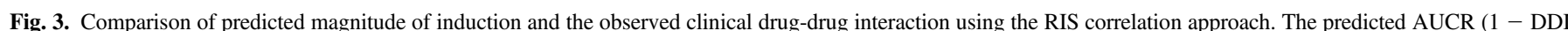

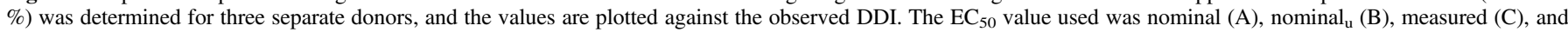

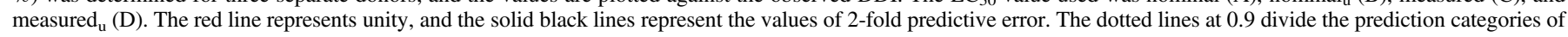
true- and false-positive and -negative inducers.

evaluated the impact of using intracellular unbound concentrations on induction predictions.

To fit an $\mathrm{EC}_{50}$ value using average unbound intracellular concentrations, the ratio of the unbound cell to unbound medium concentrations at steady state $\left(\mathrm{K}_{\text {puu,in vitro }}\right)$ needed to be determined first. An initial time- course experiment was conducted to assess which time point could be used to ensure that the $\mathrm{K}_{\text {puu, in vitro }}$ had reached steady state. Medium and intracellular concentrations were measured 0.25, 2, 4, 6, 18, and 24 hours after dosing of $1 \mu \mathrm{M}$ bosentan or $0.1 \mu \mathrm{M}$ rifampicin. Total intracellular concentrations reached a maximum by 4 (bosentan, 35-50 $\mu \mathrm{M}$ ) or 

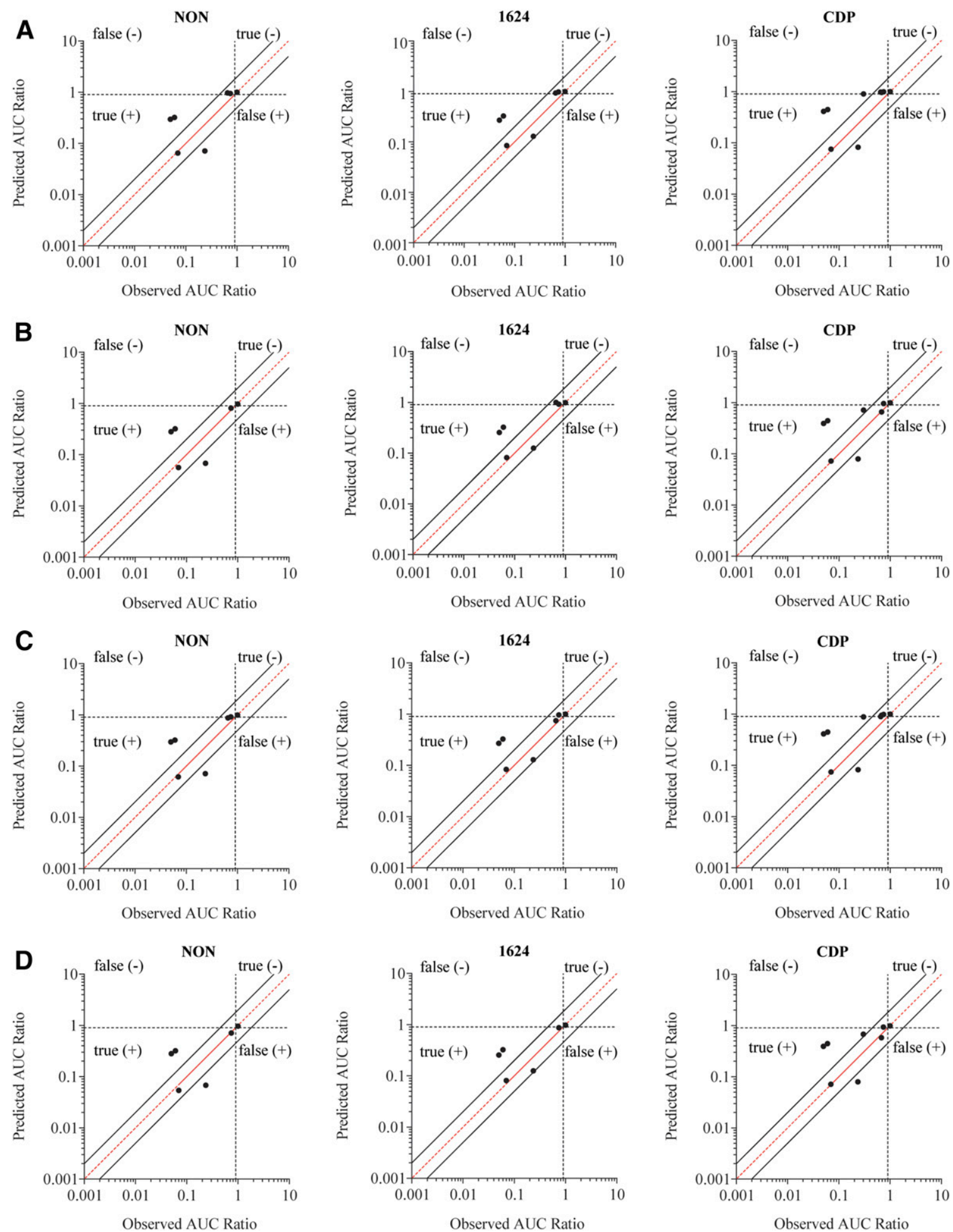

Fig. 4. Comparison of predicted magnitude of induction and the observed clinical drug-drug interaction using the basic static $\mathrm{R}_{3}$ model. The predicted AUCR was determined for three separate donors, and the values are plotted against the observed DDI. The $\mathrm{EC}_{50}$ value used was nominal $(\mathrm{A})$, nominal $_{\mathrm{u}}(\mathrm{B})$, measured $(\mathrm{C})$, and measured $\mathrm{u}_{\mathrm{u}}$ (D). The red line represents unity, and the solid black lines represent the values of 2 -fold predictive error. The dotted lines at 0.9 divide the prediction categories of true- and false-positive and -negative inducers.

6 hours (rifampicin, 5-9 $\mu \mathrm{M}$ ) prior to declining (Fig. 6). Both rifampicin $\left(f_{u, h o m}=0.194\right)$ and bosentan $\left(f_{u, h o m}=0.261\right)$ were extensively bound to hepatocyte homogenate, yielding $\mathrm{f}_{\mathrm{u}, \mathrm{cell}}$ values of 0.0096 and 0.0139 , respectively. Recovery was between 98 and 109\%, confirming compound stability over the course of the incubation. The $\mathrm{K}_{\text {puu, in vitro values }}$ were plotted as a function of time to determine the time at which $\mathrm{K}_{\text {puu,in }}$ vitro reached steady state. By 24 hours, $\mathrm{K}_{\text {puu,in vitro }}$ reached an average of 0.92 (rifampicin) and 2.9 (bosentan) at 24 hours (Fig. 6). For both bosentan and rifampicin, the $\mathrm{K}_{\text {puu,in vitro values remained steady after }}$ 18 hours (Fig. 6), so follow-up studies were performed at the 24-hour 

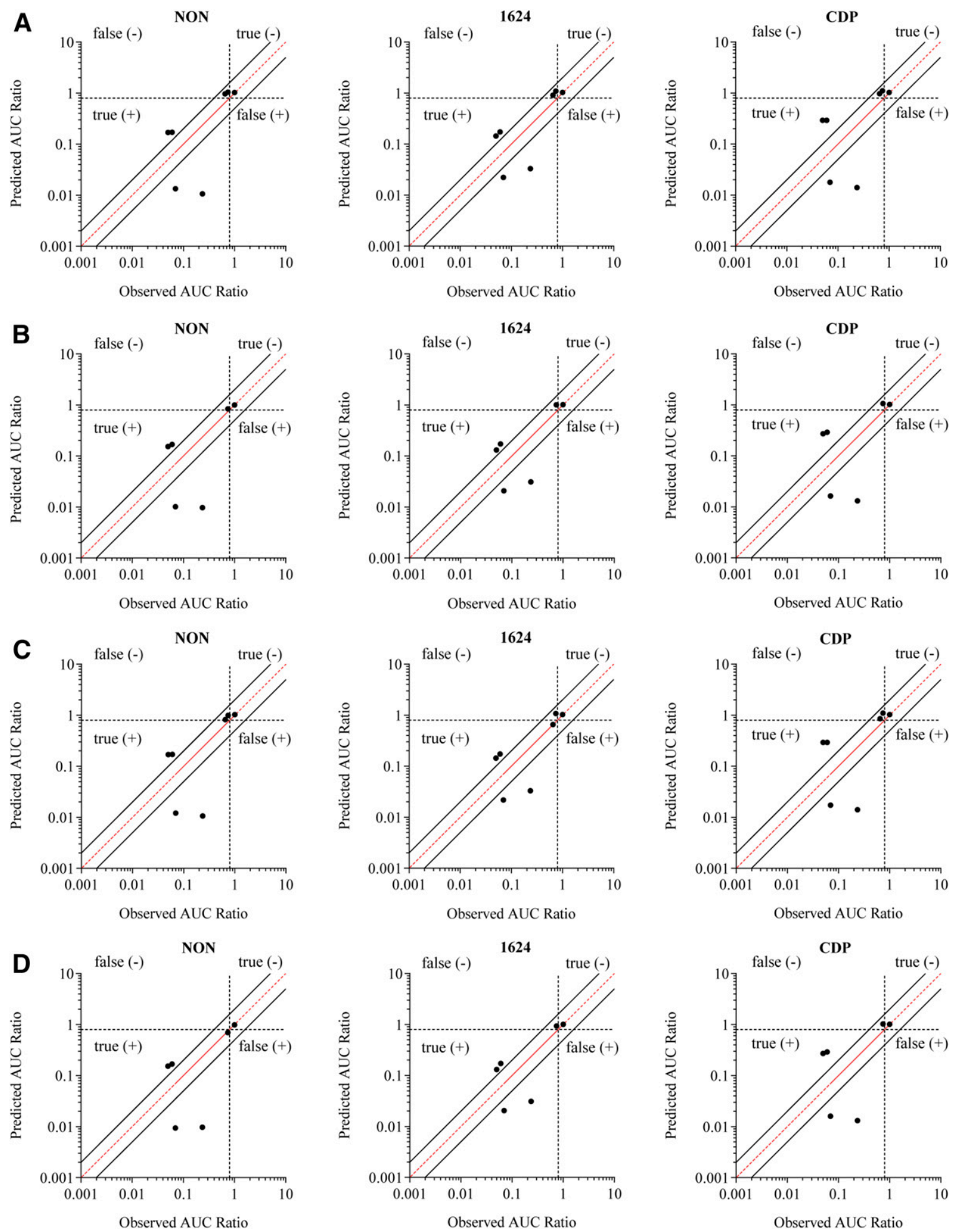

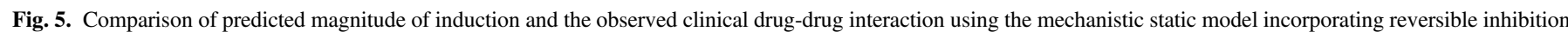

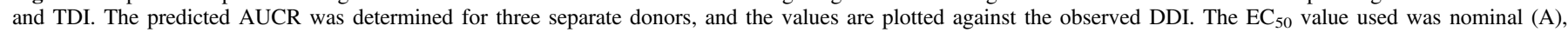

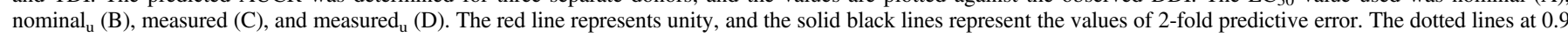
divide the prediction categories of true- and false-positive and -negative inducers.

time point. $K_{\text {puu,in vitro values can be concentration-dependent (Riccardi }}$ et al., 2016), so a follow-up study was performed in which the cell and medium concentrations were measured following incubation with the full range of inducer concentrations for 24 hours. The $\mathrm{K}_{\text {puu,in vitro values }}$ decreased as the nominal concentrations of bosentan and rifampicin increased (Fig. 6; Supplemental Table 7), likely due to saturation of uptake transporters. For each nominal inducer concentration, the average unbound medium concentrations were multiplied by the corresponding $\mathrm{K}_{\text {puu }}$ value to determine the average steady-state intracellular unbound concentration $\left(I_{u, c e l l, a v e}\right)$ (eq. 17). $I_{u, \text { cell,ave }}$ was plotted against the fold 
TABLE 3

Bosentan stability, binding, induction parameter estimates, and induction predictions

\begin{tabular}{|c|c|c|c|c|c|c|c|}
\hline Hepatocyte Lot & Average $\%$ Remaining & $\mathrm{f}_{\mathrm{u}, \text { medium }}$ & $\mathrm{E}_{\max }$ & Nominal $\mathrm{EC}_{50}$ & Measured $\mathrm{EC}_{50}$ & Nominal $\mathrm{EC}_{50, \mathrm{u}}$ & Measured $\mathrm{EC}_{50, \mathrm{u}}$ \\
\hline CDP & 33.2 & 0.38 & 9.4 & 1.6 & 0.7 & 0.6 & 0.3 \\
\hline Hu1624 & 44.1 & 0.38 & 11.6 & 3.0 & 1.0 & 1.1 & 0.4 \\
\hline \multicolumn{8}{|l|}{ Prediction method } \\
\hline \multicolumn{8}{|c|}{$\mathrm{R}_{3}$} \\
\hline $\mathrm{CDP}$ & & & $34^{a}$ & $29.1^{b}$ & $46.9^{b}$ & $50.4^{b}$ & $67.0^{b}$ \\
\hline Hu1624 & & & $34^{a}$ & $21.8^{b}$ & $44.6^{b}$ & $41.6^{b}$ & $65.7^{b}$ \\
\hline \multicolumn{8}{|l|}{ AUCR } \\
\hline $\mathrm{CDP}$ & & & $34^{a}$ & $29.4^{b}$ & $48.2^{b}$ & $51.9^{b}$ & $70.1^{b}$ \\
\hline Hu1624 & & & $34^{a}$ & $22.0^{b}$ & $45.7^{b}$ & $42.5^{b}$ & $68.6^{b}$ \\
\hline
\end{tabular}

${ }^{a}$ Observed \% DDI

${ }^{b}$ Predicted \% DDI

induction, and the resulting $\mathrm{E}_{\max , \mathrm{u}, \text { cell }}$ and $\mathrm{EC}_{50, \mathrm{u}, \text { cell }}$ are reported in Table 5. For bosentan, correction for $\mathrm{I}_{\mathrm{u}, \text { cell,ave }}$ resulted in $\mathrm{EC}_{50, \mathrm{u}, \mathrm{cell}}$ (Table 5) values that were 2.3- and 2.8-fold higher than the measured $\mathrm{EC}_{50, \mathrm{u}}$ (Table 3) in donors CDP and Hu1624, respectively. Conversely, the $\mathrm{EC}_{50, \mathrm{u}, \mathrm{cell}}$ values for rifampicin in donors CDP and Hu1624 (Table 5) were 38 and $84 \%$ of the measured $\mathrm{EC}_{50, \mathrm{u}}$ values, respectively (Table 1).

Prediction of In Vivo $\mathbf{K}_{\text {puu }} \cdot K_{\text {puu,in vivo }}$ was calculated according to eq. 20 using biliary clearance and metabolism data reported previously (Kobayashi et al., 2012; Varma et al., 2014). Active uptake and passive diffusion of bosentan and rifampicin were assessed in pooled suspension hepatocytes, and the results are reported in Table 4 and representative plots are shown in Supplemental Fig. 3. The in vivo hepatic clearance values previously reported by Loos et al. (1985) (rifampicin) and Weber et al. (1999) (bosentan) were underpredicted for both compounds, so scaling factors were applied to bosentan $(\mathrm{SF}=4.3)$ and rifampicin $(\mathrm{SF}=$ 4.1) $\mathrm{CL}_{\text {int,uptake }}$ to recover the estimated in vivo $\mathrm{CL}_{\text {int,uptake }}$ (Table 4). Based on the data in Table 4 , the $\mathrm{K}_{\text {puu,in vivo values for bosentan and }}$ rifampicin after a single dose were 4 and 15.9, respectively.

The Impact of Intracellular Unbound Inducer Concentrations on Induction Predictions. Incorporating the $\mathrm{E}_{\mathrm{max}, \mathrm{cell}}, \mathrm{EC}_{50, \mathrm{u}, \mathrm{cell}}$, and
A
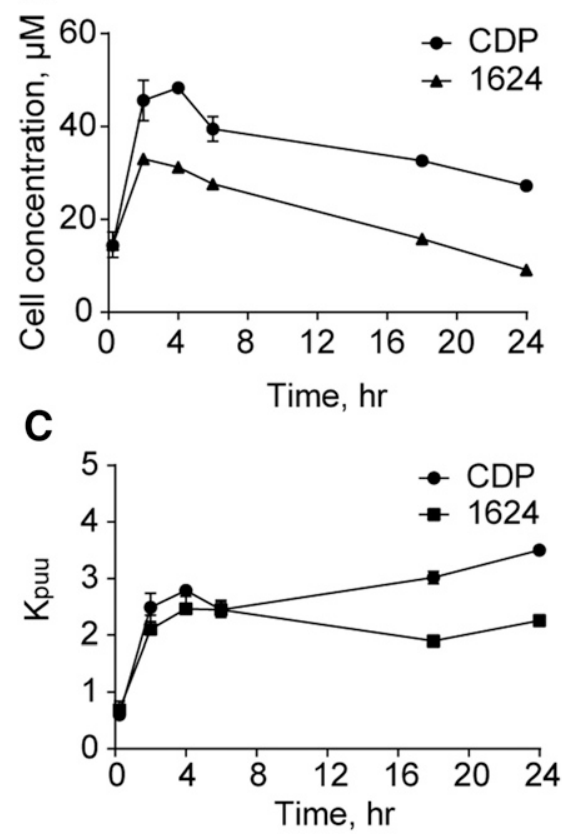

E

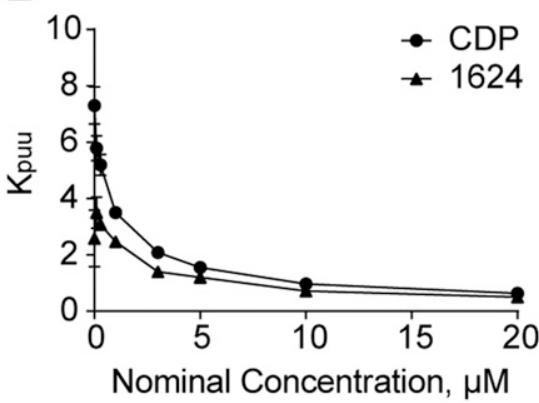

B

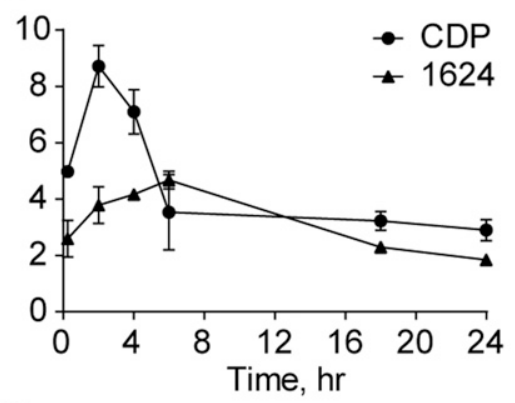

D

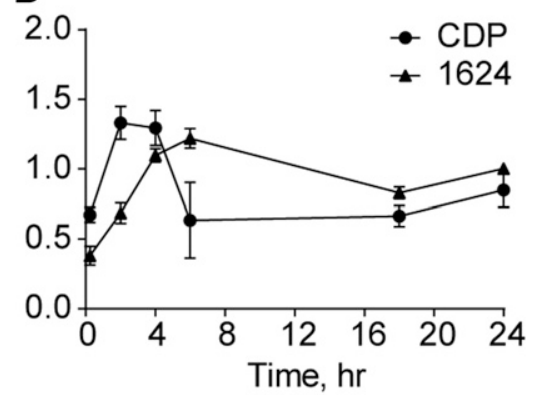

$\mathbf{F}$

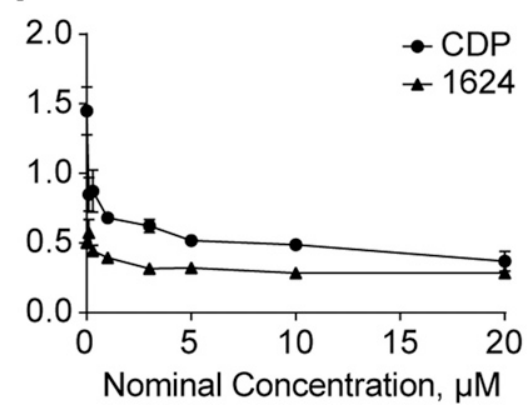

Fig. 6. Intracellular concentrations and $\mathrm{K}_{\text {puu,in vitro }}$ assessment for bosentan and rifampicin. The total intracellular concentrations of bosentan (A) and rifampicin (B) over time following incubation of cryopreserved human hepatocytes with $1 \mu \mathrm{M}$ bosentan or $0.1 \mu \mathrm{M}$ rifampicin. The $\mathrm{K}_{\mathrm{puu} \text { in vitro }}$ of bosentan $(\mathrm{C})$ and rifampicin (D) over time. The $\mathrm{K}_{\text {puu,in vitro }}$ of bosentan (E) and rifampicin (F) following incubation of multiple concentrations of bosentan or rifampicin in cryopreserved human hepatocytes for 24 hours. 
TABLE 4

Summary of input parameters for estimation of in vivo $\mathrm{K}_{\mathrm{puu}}$ for bosentan and rifampicin

\begin{tabular}{|c|c|c|c|c|c|c|c|c|c|c|c|c|}
\hline Compound & $\mathrm{CL}^{a}$ & $\mathrm{CL}_{\mathrm{R}}{ }^{a}$ & $\mathrm{f}_{\mathrm{u}, \mathrm{p}}{ }^{b}$ & $\mathrm{R}_{\mathrm{b}}^{c}$ & In Vivo $\mathrm{CL}_{\text {int,h }}$ & In Vivo $\mathrm{CL}_{\text {int,uptake }}{ }^{d}$ & $\mathrm{CL}_{\text {int,uptake }}$ & $\mathrm{CL}_{\text {int,bile }}{ }^{e}$ & $\mathrm{CL}_{\text {int,passive }}$ & $\mathrm{CL}_{\text {int,metab }}$ & $\mathrm{SF}_{\text {uptake }}$ & $\mathrm{K}_{\text {puu }}$ \\
\hline & $l / h$ & $l / h$ & & & $(\mathrm{ml} / \mathrm{min} / \mathrm{kg})$ & $\mu \mathrm{l} / \mathrm{min} / 10^{6} \mathrm{cells}$ & $\mu l / \mathrm{min} / 10^{6} \mathrm{cells}$ & $\mu l / \mathrm{min} / 10^{6} \mathrm{cells}$ & $\mu l / m i n / 10^{6} \mathrm{cells}$ & $(\mu \mathrm{l} / \mathrm{min} / \mathrm{mg})$ & & \\
\hline Bosentan & 12.3 & 0.1 & 0.037 & 0.62 & 101 & 74.0 & 17.1 & 2.0 & 13.2 & $20^{f}$ & 4.32 & 4 \\
\hline Bosentan multiple dose & 22.1 & & & & 234 & & & & & $400^{g}$ & & 0.6 \\
\hline Rifampicin & 7.5 & 1.2 & 0.15 & 0.90 & 10.9 & 50.6 & 12.3 & - & 3.1 & 0.7 & 4.11 & 15.9 \\
\hline Rifampicin multiple dose & 13 & & & & 21.9 & & & & & $1.5^{g}$ & & 14.7 \\
\hline
\end{tabular}

${ }^{a}$ Plasma clearance $(\mathrm{CL})$ and renal clearance $\left(\mathrm{CL}_{\mathrm{R}}\right)$ following an intravenous dose of bosentan (Weber et al., 1996) or rifampicin (day 2 and day 9) (Loos et al., 1985). For the induced bosentan CL following 8 days of dosing, estimated to be $22.1 \mathrm{l} / \mathrm{h}$ based on a 1.8 -fold change in oral clearance over 8 days of dosing with $200 \mathrm{mg}$ of oral bosentan (Weber et al., 1999).

${ }^{b}$ Plasma protein-binding values were reported previously for bosentan (Obach et al., 2008) and rifampicin (Varma et al., 2014).

${ }^{c}$ The blood-to-plasma ratios $\left(\mathrm{R}_{\mathrm{b}}\right)$ were reported previously (Varma et al., 2014).

${ }^{d}$ The in vivo estimate of $\mathrm{CL}_{\text {int, uptake }}$ was calculated from the in vivo $\mathrm{CL}_{\text {int,h }}$ using the $\mathrm{CL}_{\text {int,passive }}, \mathrm{CL}_{\text {int,bile, and }} \mathrm{CL}_{\text {int,metab }}$ listed in the table.

${ }^{e} \mathrm{CL}_{\text {int,bile }}$ for bosentan was reported by Varma et al. (2014).

${ }^{f} \mathrm{CL}_{\text {int,metab }}$ for bosentan was reported by Varma et al. (2014).

${ }^{g} \mathrm{CL}_{\text {int,metab }}$ was calculated from the multiple-day bosentan and rifampicin in vivo $\mathrm{CL}_{\text {int, } \mathrm{h}}$, assuming $\mathrm{CL}_{\text {int }}$, uptake, $\mathrm{CL}_{\text {int,bile }}$, and $\mathrm{CL}_{\text {int,passive }}$ remained constant.

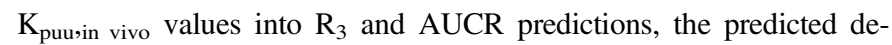
creases in simvastatin exposure were on average 76.5 and 76.8 , respectively (Table 5). The magnitude of induction predicted using this method resulted in a higher degree of overprediction of bosentan than what was predicted using measured $\mathrm{EC}_{50, \mathrm{u}}$ and $\mathrm{C}_{\text {max,u }}$ values (Tables 3 and 5). For rifampicin, the magnitude of induction using the $\mathrm{R}_{3}$ model was predicted to be roughly equivalent (93\% DDI) regardless of whether $\mathrm{E}_{\max , \text { cell }}, \mathrm{EC}_{50 \text {, } \mathrm{u}, \mathrm{cell}}$, and $\mathrm{K}_{\text {puu, in vivo }}$ or $\mathrm{E}_{\mathrm{max}}, \mathrm{EC}_{50, \mathrm{u}}$, and $\mathrm{C}_{\text {max,u }}$ were used (Tables 1 and 5). However, $\mathrm{E}_{\text {max,cell }}$ and $\mathrm{EC}_{50, \mathrm{u}, \mathrm{cell}}$, resulted in a slightly lower predicted magnitude of DDI (96\%) than when $\mathrm{E}_{\max }, \mathrm{EC}_{50, \mathrm{u}}$, and $\mathrm{C}_{\max , \mathrm{u}}$ were used (98\%).

The limitation to using single-dose clearance data to predict $\mathrm{K}_{\text {puu,in vitro }}$ is that they do not account for reported autoinduction of hepatic clearance for bosentan and rifampicin (Loos et al., 1985; Weber et al., 1999). To estimate $\mathrm{K}_{\text {puu,in vivo }}$ after multiple doses, a new value for $\mathrm{CL}_{\text {int,metab }}$ was calculated (Table 4) based on multiple-dose $\mathrm{CL}_{\mathrm{h}}$ (Loos et al., 1985; Weber et al., 1999) assuming no other elimination pathways were induced. The calculated $\mathrm{CL}_{\mathrm{int}}$, metab value was then incorporated into eq. 20 to calculate $\mathrm{K}_{\text {puu,invivo }}$ following multiple doses. The resulting $\mathrm{K}_{\text {puu,invivo }}$ values were 0.59 and 14.7 for bosentan and rifampicin, respectively (Table 4). Using the multiple-dose $\mathrm{K}_{\text {puu,in vivo, }}$ the predicted magnitude of induction for bosentan using both the $\mathrm{R}_{3}(38.5 \% \mathrm{DDI})$ and AUCR (41\% DDI) models was in good agreement with the observed $34 \%$ DDI (Table 5). However, for rifampicin, predictions using multiple-dose $\mathrm{K}_{\text {puu,in vivo }}$ versus single-dose $\mathrm{K}_{\text {puu,in vivo }}$ were identical.

\section{Discussion}

Although cultured human hepatocytes are considered the "gold standard" in vitro system with which to assess CYP3A4 induction risk in the clinic (Chu et al., 2009), the dynamics of the system are not always accounted for, and DDI risk is generally based upon nominal concentrations of perpetrators (Almond et al., 2009). Furthermore, BSA (0-2\%) is typically added to cell culture medium (Runge et al., 2000; Madan et al., 2003; Nishimura et al., 2007) as a supplement to increase overall cell health, block nonspecific binding sites, and improve the solubility of compounds with poor chemical properties (Francis, 2010). One caveat of including BSA in induction medium is that it can lower the concentration of free drug. Although inducer depletion and binding have previously been accounted for in induction predictions (Zhang et al., 2014; Chang et al., 2016), to date, no comprehensive study has been conducted to systematically investigate the impact of binding and depletion on induction predictions across multiple inducers and prediction methods. In the current evaluation, we sought to determine the measured and free concentrations of six clinical inducers and one noninducer of CYP3A4 in hepatocyte culture medium, and determine whether correction for these parameters can improve the accuracy of CYP3A4 induction predictions.

Medium concentrations were measured over the course of a dosing interval to assess the effects of nonspecific binding and metabolism of the seven compounds used in our study (Fig. 1). Parallel studies were conducted to assess the impact of protein binding on medium supplements in our studies (Table 1). After quantifying the perpetrator concentrations in the induction medium, various modeling approaches were used to assess whether correcting for measured and free concentration of perpetrator improve the accuracy and precision of the predictions. To evaluate prediction success, predictions were categorized as false-positive, falsenegative, true-positive, or true-negative, and the accuracy and bias were assessed using RMSE and GMFE (Table 2). With respect to categorical predictions, the RIS model was the most reliable, resulting in no false-positive or false-negative predictions. Only two out of seven predictions gave a false-negative prediction when nominal $\mathrm{EC}_{50}$ values were used in each lot of hepatocytes with the $\mathrm{R}_{3}$ and mechanistic static predictions. The percentage of false negatives was reduced when measured $\mathrm{EC}_{50}$ values were used and were further reduced when unbound $\mathrm{EC}_{50}$ values were used. Similarly, the RIS model had the greatest accuracy and least bias, as evidenced by low RMSE and GMFE values, whereas the mechanistic static model had the highest values. After the RIS model, the mechanistic static model resulted in the greatest prediction accuracy. However, predicted induction magnitude using this model was higher for all of the compounds aside from pioglitazone and rosiglitazone (Supplemental Tables 4-6), and rifampicin induction was actually overpredicted. This trend toward overprediction of CYP3A4 induction is likely due to the incorporation of intestinal induction into the mechanistic static model. This suggests that the mechanistic static model may only be advantageous when an inducer also causes reversible and/or time-dependent inhibition. Across all of the models, the accuracy of the predictions was greatest when both binding and medium concentrations were considered.

The observation that measured $\mathrm{EC}_{50}$ values resulted in more accurate predictions is in contrast to findings from Zhang et al. (2014) who measured medium concentrations of 20 compounds at the 24-hour time point on the last day of incubation. For six compounds, measured concentrations of the perpetrator were $<20 \%$ of the nominal concentrations. For these six compounds, the authors determined the average concentration by taking the average of the nominal and measured 24-hour concentrations. The $\mathrm{EC}_{50}$ 
TABLE 5

Rifampicin and bosentan induction predictions incorporating $\mathrm{K}_{\text {puu }}$

\begin{tabular}{|c|c|c|c|c|c|}
\hline \multirow[b]{2}{*}{$\begin{array}{l}\text { Hepatocyte Lot } \\
\text { Bosentan }\end{array}$} & \multirow[b]{2}{*}{$\mathrm{E}_{\mathrm{max}, \mathrm{u}, \mathrm{cell}}$} & \multirow[b]{2}{*}{$\mathrm{EC}_{50, \mathrm{u}, \mathrm{cell}}$} & \multicolumn{2}{|c|}{ Predicted \% DDI } & \multirow{2}{*}{ Observed \% DDI } \\
\hline & & & R3 & AUCR & \\
\hline CDP & 8.8 & 0.7 & 72.6 & 72.9 & 34 \\
\hline $\mathrm{Hu} 1624$ & 18.6 & 1.1 & 80.3 & 80.6 & 34 \\
\hline \multicolumn{6}{|c|}{ Bosentan multiple dose } \\
\hline CDP & 8.8 & 0.7 & 34.6 & 36.73 & 34 \\
\hline $\mathrm{Hu} 1624$ & 18.6 & 1.1 & 42.5 & 45.2 & 34 \\
\hline \multicolumn{6}{|l|}{ Rifampicin } \\
\hline CDP & 14.3 & 0.16 & 93.4 & 96.2 & 93 \\
\hline $\mathrm{Hu} 1624$ & 12.4 & 0.058 & 92.5 & 95.4 & 93 \\
\hline \multicolumn{6}{|c|}{ Rifampicin multiple dose } \\
\hline CDP & 14.3 & 0.16 & 93.4 & 96.2 & 93 \\
\hline $\mathrm{Hu} 1624$ & 12.4 & 0.058 & 92.5 & 95.4 & 93 \\
\hline
\end{tabular}

values were then corrected by using the estimated average medium concentration. However, correction of the $\mathrm{EC}_{50}$ values for the six compounds did not improve the RMSE and GMFE for any parameter. One possible explanation for this discrepancy is that nonspecific binding was not considered. By estimating the average medium concentration from the average of the nominal and 24-hour concentrations, one assumes that the loss of compound from the medium is due to linear depletion. However, extensive nonspecific binding to the cells or plate could result in an immediate reduction in medium concentration, as was observed for pleconaril in the present study, where only $\sim 8 \%$ remained after a 24 -hour incubation period (Table 1). The significant loss of pleconaril in the induction medium was observed at both $4^{\circ} \mathrm{C}$ and $37^{\circ} \mathrm{C}$, with a greater loss observed at $37^{\circ} \mathrm{C}$, indicating that the loss of pleconaril may be caused by both metabolism and nonspecific binding (to cell plates and/or hepatocytes). In such a case, the method used by Zhang et al. (2014) would be expected to overestimate the average medium concentration and underestimate the reduction in the $\mathrm{EC}_{50}$, potentially preventing prediction improvements. A second potential reason why the use of calculated average medium concentrations may not have improved predictions in the studies by Zhang et al. (2014) is that medium protein binding was not accounted for. The six compounds with low medium recovery (troglitazone, terbinafine, pleconaril, omeprazole, clotrimazole, and nifedipine) are highly bound in plasma.

In addition to accounting for depletion and binding in the determination of in vitro induction parameters, we hypothesized that estimating $\mathrm{E}_{\max }$ and $\mathrm{EC}_{50}$ values based on unbound intracellular concentrations may improve predictions for CYP3A4 inducers that are transporter substrates. Bosentan and rifampicin are both permeabilitylimited substrates of OATP1B1 and OATP1B3, so the ratio of unbound intracellular concentrations to unbound medium concentrations $\left(\mathrm{K}_{\text {puu,in vitro }}\right)$ cannot be assumed to equal 1 . Bosentan induction was overpredicted by roughly 2 -fold when measured $\mathrm{EC}_{50, \mathrm{u}}$ and $\mathrm{C}_{\max , \mathrm{u}}$ values were used in predictions. We hypothesized that this was due to free cell concentrations exceeding free medium concentrations. As a result, $\mathrm{EC}_{50}$ values based on free medium concentrations would overestimate the true induction potency. In the current study, a concentration-dependent range of $\mathrm{K}_{\text {puu,in vitro }}$ values was observed for rifampicin (0.3-1.5) and bosentan (0.5-7.5) across the inducer concentrations used to fit the $\mathrm{E}_{\mathrm{max}, \mathrm{u}, \mathrm{cell}}$ and $\mathrm{EC}_{50, \mathrm{u}, \text { cell }}$. Fitting the $\mathrm{E}_{\text {max,u,cell }}$ and $\mathrm{EC}_{50, \mathrm{u}, \mathrm{cell}}$ resulted in $\mathrm{EC}_{50}$ values that were 2.6 (bosentan) and 0.6 (rifampicin) times the measured $\mathrm{EC}_{50, \mathrm{u}}$. The higher $\mathrm{EC}_{50, \mathrm{u}, \mathrm{cell}}$ estimate was consistent with the hypothesis that the measured $\mathrm{EC}_{50, \mathrm{u}}$ overestimated bosentan induction potency, and resulted in improved predictions for bosentan when the predicted multiple-dose $\mathrm{K}_{\text {puu,in vivo }}(0.6)$ was used, which accounted for autoinduction of bosentan metabolism. Unlike bosentan, rifampicin predictions were not dramatically affected by corrections for binding, depletion, or intracellular concentrations. The reason for the lack of change for rifampicin predictions is because, in all cases, the in vivo inducer concentration used in the predictions greatly exceeded the $\mathrm{EC}_{50}(>10$ fold), and thus the predicted fold induction was equivalent to $1+\mathrm{E}_{\max }$.

To date, numerous methods have been proposed for determining intracellular unbound concentrations of drugs; however, as highlighted in a recent white paper, no systematic evaluation has been conducted to determine which method is the most reliable (Ulvestad et al., 2011; Zhu et al., 2014). Estimation of the $f_{u, \text { cell }}$ using cell homogenate has been widely used in recent years (Mateus et al., 2013; Chien et al., 2016; Riccardi et al., 2016). Furthermore, the $K_{\text {puu,in vitro values estimated }}$ using this method were reported to be in agreement with the fold difference in $\mathrm{IC}_{50}$ estimated in human liver microsomes versus cell lines, providing confidence in the estimated $\mathrm{f}_{\mathrm{u}, \text { cell }}$ (Riccardi et al., 2016). However, a major limitation of this method is that it does not account for sequestration of drug into subcellular organelles (Chu et al., 2013; Kazmi et al., 2013). Because rifampicin and bosentan are acids, $\mathrm{pH}$-driven lysosomal and mitochondrial trapping is not anticipated in the present study but may be a deterrent for using the homogenization approach to determine $\mathrm{f}_{\mathrm{u} \text {,cell }}$ for weak bases (Duvvuri et al., 2004; Ufuk et al., 2015).

Use of static models to predict $\mathrm{K}_{\text {puu,in vivo }}$ (Barton et al., 2013; Varma et al., 2014) also poses limitations for the performance of predictions. Time-dependent changes in metabolism and transport can affect the $\mathrm{K}_{\text {puu,in vivo }}$ estimate, and are not captured by static models. In this study, the $K_{\text {puu,in vivo }}$ after multiple days of bosentan or rifampicin treatment was estimated by adjusting the $\mathrm{CL}_{\text {int,metab }}$ so that the $\mathrm{CL}_{\text {int,h }}$ matched the in vivo $\mathrm{CL}_{\text {int,h }}$ following multiple doses (Loos et al., 1985; Weber et al., 1999). However, this does not capture the time-dependent changes in

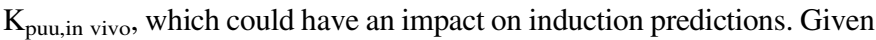
the limitation of this approach, efforts are ongoing in our laboratory to assess the impact of the $\mathrm{K}_{\text {puu }}$ estimates using dynamic mechanistic models rather than the static models.

In conclusion, data indicate that quantitating NCE levels in induction medium by correcting for specific binding to medium proteins, nonspecific binding, and measured medium concentrations $\left(\mathrm{C}_{\text {ave }}\right)$ can improve the accuracy and precision of CYP3A4 induction predictions. Further work needs to be conducted to understand the impact of free intracellular concentrations $\left(\mathrm{K}_{\text {puu }}\right)$ of NCEs on predictions when compounds are known to be substrates of uptake transporters and inducers of CYP3A4. 


\section{Authorship Contributors}

Participated in research design: Sun, Chothe, Sager, Tsao, Moore, Laitinen, Hariparsad.

Conducted experiments: Sun, Chothe, Sager, Tsao, Moore, Laitinen.

Performed data analysis: Sun, Chothe, Sager, Tsao, Moore, Laitinen.

Wrote or contributed to the writing of the manuscript: Sun, Chothe, Sager, Tsao, Moore, Laitinen, Hariparsad.

\section{References}

Almond LM, Yang J, Jamei M, Tucker GT, and Rostami-Hodjegan A (2009) Towards a quantitative framework for the prediction of DDIs arising from cytochrome P450 induction. Curr Drug Metab 10:420-432.

Barton HA, Lai Y, Goosen TC, Jones HM, El-Kattan AF, Gosset JR, Lin J, and Varma MV (2013) Model-based approaches to predict drug-drug interactions associated with hepatic uptake transporters: preclinical, clinical and beyond. Expert Opin Drug Metab Toxicol 9:459-472.

Chang C, Yang X, Fahmi OA, Riccardi KA, Di L, and Obach RS (2016) An exposure-response analysis based on rifampin suggests CYP3A4 induction is driven by AUC: an in vitro investigation. Xenobiotica $1-9$.

Chien HC, Zur AA, Maurer TS, Yee SW, Tolsma J, Jasper P, Scott DO, and Giacomini KM (2016) Rapid Method To Determine Intracellular Drug Concentrations in Cellular Uptake Assays: Application to Metformin in Organic Cation Transporter 1-Transfected Human Embryonic Kidney 293 Cells. Drug Metab Dispos 44:356-364.

Chu V, Einolf HJ, Evers R, Kumar G, Moore D, Ripp S, Silva J, Sinha V, Sinz M, and Skerjanec A (2009) In vitro and in vivo induction of cytochrome p450: a survey of the current practices and recommendations: a pharmaceutical research and manufacturers of america perspective. Drug Metab Dispos 37:1339-1354.

Chu X, Korzekwa K, Elsby R, Fenner K, Galetin A, Lai Y, Matsson P, Moss A, Nagar S, Rosania GR, et al.; International Transporter Consortium (2013) Intracellular drug concentrations and transporters: measurement, modeling, and implications for the liver. Clin Pharmacol Ther 94:126-141.

Chung E, Nafziger AN, Kazierad DJ, and Bertino, Jr JS (2006) Comparison of midazolam and simvastatin as cytochrome P450 3A probes. Clin Pharmacol Ther 79:350-361.

Duvvuri M, Gong Y, Chatterji D, and Krise JP (2004) Weak base permeability characteristics influence the intracellular sequestration site in the multidrug-resistant human leukemic cell line HL-60. J Biol Chem 279:32367-32372.

Einolf HJ, Chen L, Fahmi OA, Gibson CR, Obach RS, Shebley M, Silva J, Sinz MW, Unadkat JD, Zhang L, et al. (2014) Evaluation of various static and dynamic modeling methods to predict clinical CYP3A induction using in vitro CYP3A4 mRNA induction data. Clin Pharmacol Ther 95:179-188.

Fahmi OA, Boldt S, Kish M, Obach RS, and Tremaine LM (2008) Prediction of drug-drug interactions from in vitro induction data: application of the relative induction score approach using cryopreserved human hepatocytes. Drug Metab Dispos 36:1971-1974.

Fahmi OA, Hurst S, Plowchalk D, Cook J, Guo F, Youdim K, Dickins M, Phipps A, Darekar A, Hyland R, et al. (2009) Comparison of different algorithms for predicting clinical drug-drug interactions, based on the use of CYP3A4 in vitro data: predictions of compounds as precipitants of interaction. Drug Metab Dispos 37:1658-1666.

Francis GL (2010) Albumin and mammalian cell culture: implications for biotechnology applications. Cytotechnology 62:1-16.

Galetin A, Burt H, Gibbons L, and Houston JB (2006) Prediction of time-dependent CYP3A4 drug-drug interactions: impact of enzyme degradation, parallel elimination pathways, and intestinal inhibition. Drug Metab Dispos 34:166-175.

Izumi S, Nozaki Y, Maeda K, Komori T, Takenaka O, Kusuhara H, and Sugiyama Y (2015) Investigation of the impact of substrate selection on in vitro organic anion transporting polypeptide 1B1 inhibition profiles for the prediction of drug-drug interactions. Drug Metab Dispos 43:235-247.

Kato M, Chiba K, Horikawa M, and Sugiyama Y (2005) The quantitative prediction of in vivo enzyme-induction caused by drug exposure from in vitro information on human hepatocytes. Drug Metab Pharmacokinet 20:236-243.

Kazmi F, Hensley T, Pope C, Funk RS, Loewen GJ, Buckley DB, and Parkinson A (2013) Lysosomal sequestration (trapping) of lipophilic amine (cationic amphiphilic) drugs in immortalized human hepatocytes (Fa2N-4 cells). Drug Metab Dispos 41:897-905.

Kobayashi Y, Fukami T, Nakajima A, Watanabe A, Nakajima M, and Yokoi T (2012) Species differences in tissue distribution and enzyme activities of arylacetamide deacetylase in human, rat, and mouse. Drug Metab Dispos 40:671-679.

Kozawa M, Honma M, and Suzuki H (2009) Quantitative prediction of in vivo profiles of CYP3A4 induction in humans from in vitro results with a reporter gene assay. Drug Metab Dispos 37 $1234-1241$.

Lin JH (2006) CYP induction-mediated drug interactions: in vitro assessment and clinical implications. Pharm Res 23:1089-1116.

Liu L and Pang KS (2005) The roles of transporters and enzymes in hepatic drug processing. Drug Metab Dispos 33:1-9.

Loos U, Musch E, Jensen JC, Mikus G, Schwabe HK, and Eichelbaum M (1985) Pharmacokinetics of oral and intravenous rifampicin during chronic administration. Klin Wochenschr 63 $1205-1211$.

Madan A, Graham RA, Carroll KM, Mudra DR, Burton LA, Krueger LA, Downey AD, Czerwinski M, Forster J, Ribadeneira MD, et al. (2003) Effects of prototypical microsomal enzyme inducers on cytochrome P450 expression in cultured human hepatocytes. Drug Metab Dispos 31:421-431.
Mateus A, Matsson P, and Artursson P (2013) Rapid measurement of intracellular unbound drug concentrations. Mol Pharm 10:2467-2478.

Nishimura M, Koeda A, Suganuma Y, Suzuki E, Shimizu T, Nakayama M, Satoh T, Narimatsu S, and Naito S (2007) Comparison of inducibility of CYP1A and CYP3A mRNAs by prototypical inducers in primary cultures of human, cynomolgus monkey, and rat hepatocytes. Drug Metab Pharmacokinet 22:178-186.

Obach RS, Lombardo F, and Waters NJ (2008) Trend analysis of a database of intravenous pharmacokinetic parameters in humans for 670 drug compounds. Drug Metab Dispos 36: $1385-1405$.

Obach RS, Walsky RL, Venkatakrishnan K, Gaman EA, Houston JB, and Tremaine LM (2006) The utility of in vitro cytochrome $\mathrm{P} 450$ inhibition data in the prediction of drug-drug interactions. $J$ Pharmacol Exp Ther 316:336-348.

O'Brien Z and Fallah Moghaddam M (2013) Small molecule kinase inhibitors approved by the FDA from 2000 to 2011: a systematic review of preclinical ADME data. Expert Opin Drug Metab Toxicol 9:1597-1612.

Pang KS and Rowland M (1977) Hepatic clearance of drugs. I. Theoretical considerations of a "well-stirred" model and a "parallel tube" model. Influence of hepatic blood flow, plasma and blood cell binding, and the hepatocellular enzymatic activity on hepatic drug clearance. $J$ Pharmacokinet Biopharm 5:625-653.

Rebeski DE, Winger EM, Shin YK, Lelenta M, Robinson MM, Varecka R, and Crowther JR (1999) Identification of unacceptable background caused by non-specific protein adsorption to the plastic surface of 96-well immunoassay plates using a standardized enzyme-linked immunosorbent assay procedure. J Immunol Methods 226:85-92.

Reinoso RF, Telfer BA, Brennan BS, and Rowland M (2001) Uptake of teicoplanin by isolated rat hepatocytes: comparison with in vivo hepatic distribution. Drug Metab Dispos 29:453-459.

Riccardi K, Li Z, Brown JA, Gorgoglione MF, Niosi M, Gosset J, Huard K, Erion DM, and Di L (2016) Determination of unbound partition coefficient and in vitro-in vivo extrapolation for SLC13A transporter-mediated uptake. Drug Metab Dispos 44:1633-1642.

Ripp SL, Mills JB, Fahmi OA, Trevena KA, Liras JL, Maurer TS, and de Morais SM (2006) Use of immortalized human hepatocytes to predict the magnitude of clinical drug-drug interactions caused by CYP3A4 induction. Drug Metab Dispos 34:1742-1748.

Runge D, Köhler C, Kostrubsky VE, Jäger D, Lehmann T, Runge DM, May U, Stolz DB, Strom SC, Fleig WE, et al. (2000) Induction of cytochrome P450 (CYP)1A1, CYP1A2, and CYP3A4 but not of CYP2C9, CYP2C19, multidrug resistance (MDR-1) and multidrug resistance associated protein (MRP-1) by prototypical inducers in human hepatocytes. Biochem Biophys Res Commun 273:333-341.

Shou M, Hayashi M, Pan Y, Xu Y, Morrissey K, Xu L, and Skiles GL (2008) Modeling, prediction, and in vitro in vivo correlation of CYP3A4 induction. Drug Metab Dispos 36: 2355-2370.

Tirona RG, Leake BF, Wolkoff AW, and Kim RB (2003) Human organic anion transporting polypeptide-C (SLC21A6) is a major determinant of rifampin-mediated pregnane $\mathrm{X}$ receptor activation. $J$ Pharmacol Exp Ther 304:223-228.

Treiber A, Schneiter R, Häusler S, and Stieger B (2007) Bosentan is a substrate of human OATP1B1 and OATP1B3: inhibition of hepatic uptake as the common mechanism of its interactions with cyclosporin A, rifampicin, and sildenafil. Drug Metab Dispos 35:1400-1407.

Ufuk A, Somers G, Houston JB, and Galetin A (2015) In Vitro Assessment of Uptake and Lysosomal Sequestration of Respiratory Drugs in Alveolar Macrophage Cell Line NR8383. Pharm Res 32:3937-3951.

Ulvestad M, Björquist P, Molden E, Asberg A, and Andersson TB (2011) OATP1B1/1B3 activity in plated primary human hepatocytes over time in culture. Biochem Pharmacol 82:1219-1226.

Varma MV, Bi YA, Kimoto E, and Lin J (2014) Quantitative prediction of transporter- and enzyme-mediated clinical drug-drug interactions of organic anion-transporting polypeptide 1B1 substrates using a mechanistic net-effect model. J Pharmacol Exp Ther 351:214-223.

Vavricka SR, Van Montfoort J, Ha HR, Meier PJ, and Fattinger K (2002) Interactions of rifamycin SV and rifampicin with organic anion uptake systems of human liver. Hepatology 36:164-172.

Vieira ML, Kirby B, Ragueneau-Majlessi I, Galetin A, Chien JY, Einolf HJ, Fahmi OA, Fischer V, Fretland A, Grime K, et al. (2014) Evaluation of various static in vitro-in vivo extrapolation models for risk assessment of the CYP3A inhibition potential of an investigational drug. Clin Pharmacol Ther 95:189-198.

Weber C, Schmitt R, Birnboeck H, Hopfgartner G, Eggers H, Meyer J, van Marle S, Viischer HW, and Jonkman JH (1999) Multiple-dose pharmacokinetics, safety, and tolerability of bosentan, an endothelin receptor antagonist, in healthy male volunteers. J Clin Pharmacol 39:703-714.

Weber C, Schmitt R, Birnboeck H, Hopfgartner G, van Marle SP, Peeters PA, Jonkman JH, and Jones CR (1996) Pharmacokinetics and pharmacodynamics of the endothelin-receptor antagonist bosentan in healthy human subjects. Clin Pharmacol Ther 60:124-137.

Zhang JG, Ho T, Callendrello AL, Clark RJ, Santone EA, Kinsman S, Xiao D, Fox LG, Einolf HJ, and Stresser DM (2014) Evaluation of calibration curve-based approaches to predict clinical inducers and noninducers of CYP3A4 with plated human hepatocytes. Drug Metab Dispos 42 $1379-1391$.

Zhu Q, Xia H, Xia CQ, Yang Q, Doshi U, Li AP, and Liao M (2014) Culture duration-, donor-, and medium-dependent changes in OATP1B3-mediated telmisartan uptake in human hepatocytes. Drug Metab Lett 7:117-125.

Address correspondence to: Dr. Niresh Hariparsad, Department of Drug Metabolism and Pharmacokinetics, 4022 Vertex 1, 50 Northern Avenue, Boston, MA 02210. E-mail: Niresh_hariparsad@vrtx.com 